\title{
Full Characterization of Parikh's Relevance-Sensitive Axiom for Belief Revision
}

\author{
Theofanis I. Aravanis \\ Pavlos Peppas \\ Department of Business Administration \\ University of Patras \\ Patras 265 00, Greece \\ Mary-Anne Williams \\ Centre for Artificial Intelligence \\ FEIT, University of Technology Sydney \\ NSW 2007, Australia
}

MARY-ANnE.Williams@uts.EDU.AU

\begin{abstract}
In this article, the epistemic-entrenchment and partial-meet characterizations of Parikh's relevance-sensitive axiom for belief revision, known as axiom $(\mathrm{P})$, are provided. In short, axiom (P) states that, if a belief set $K$ can be divided into two disjoint compartments, and the new information $\varphi$ relates only to the first compartment, then the revision of $K$ by $\varphi$ should not affect the second compartment. Accordingly, we identify the subclass of epistemic-entrenchment and that of selection-function preorders, inducing AGM revision functions that satisfy axiom $(\mathrm{P})$. Hence, together with the faithful-preorders characterization of $(\mathrm{P})$ that has already been provided, Parikh's axiom is fully characterized in terms of all popular constructive models of Belief Revision. Since the notions of relevance and local change are inherent in almost all intellectual activity, the completion of the constructive view of $(\mathrm{P})$ has a significant impact on many theoretical, as well as applied, domains of Artificial Intelligence.
\end{abstract}

\section{Introduction}

Belief Revision is the study of knowledge dynamics. The article that is widely considered to have marked the birth of the field is the seminal work of Alchourrón, Gärdenfors, and Makinson (1985). From this work arose a formal framework, now known as the AGM paradigm, which is to date the dominant framework for the study of belief revision. Within this framework, the beliefs of an agent are modelled as a logical theory $K$, also called a belief set, epistemic input is represented as a logical sentence $\varphi$, and the revision of $K$ by $\varphi$, denoted $K * \varphi$, is modelled as a function $*$ mapping theories and sentences to theories. Revision functions are constrained by eight postulates, the AGM postulates for revision, introduced to capture the notion of rationality in the context of belief change. The functions that satisfy these postulates are known as the AGM revision functions. ${ }^{1}$

Parikh (1999) pointed out that the AGM postulates for revision are too liberal in their treatment of the notion of relevance. According to Parikh, a rational agent does not change her entire belief corpus during belief revision, but only the part of it that is relevant to

1. To distinguish the research area from the process, we shall use the capitalized term "Belief Revision" for the former, and the same term in lower-case letters (i.e., "belief revision") for the latter. 
the new information. To fully capture this intuition of local change, Parikh proposed an additional axiom, named $(\mathrm{P})$, based on a syntax-splitting approach.

It is worth pausing at this point and noting that the problem of relevance is a crucial topic in many areas of Artificial Intelligence, and, as a consequence, has received considerable attention (Subramanian, Greiner, \& Judea Pearl, 1997). Confined to relevance-sensitive belief change, reference is indicatively made to the work of Gärdenfors (1990), Nebel (1999), Chopra and Parikh (2000), Chopra, Georgatos, and Parikh (2001), Kourousias and Makinson (2007), Makinson (2009), Wu and Zhang (2008), Perrussel, Marchi, and Zhang (2010), Parikh (2011), Delgrande and Peppas (2018). An interesting application of local change for diagnosis (i.e., the process of finding the faulty compartment of a malfunctioning system) has been proposed by Wassermann (2001a), while Hansson and Wassermann (2002) used local change for handling local inconsistencies that actual agents in the real world hold. Lastly, Riana and Wassermann (2004) conducted an empirical study using relevance to cut the search space for a problem solution.

Continuing, axiom (P) was further analysed by Peppas, Williams, Chopra, and Foo (2015), where it was shown that it is open to two different interpretations; i.e., the weak (condition $(\mathrm{P} 1)$ ) and the strong (conditions $(\mathrm{P} 1)$ and $(\mathrm{P} 2)$ ) version of axiom $(\mathrm{P}){ }^{2}$ In the same work, both versions were characterized in terms of faithful preorders (a special kind of preorders over possible worlds).

In this article, we introduce such a characterization in the realm of the epistemicentrenchment and partial-meet models. In particular, we formulate new constraints on preorders over sentences of the underlying language, as well as on preorders over maximal subsets of a theory not implying the new information (remainders), that characterize precisely the class of epistemic-entrenchment and that of selection-function preorders, respectively, corresponding to AGM revision functions satisfying axiom $(\mathrm{P})$. With these results, Parikh's relevance-sensitive axiom is fully characterized in terms of all popular constructive models of Belief Revision; thus, the constructive view of axiom $(\mathrm{P})$ is completed.

The rest of the article is organized as follows: The next section introduces notation and terminology. Then, the necessary background on the AGM paradigm and the notion of relevance in Belief Revision are given. Subsequently, and to make the article self-contained, the faithful-preorders characterization of axiom (P) is presented, as introduced by Peppas et al. (2015), and, following that, the epistemic-entrenchment and partial-meet characterizations of $(\mathrm{P})$ are provided. The last section is devoted to some concluding remarks.

\section{Formal Preliminaries}

Throughout this article, we work with a finite, non-empty set of propositional variables $\mathcal{P}$. We define $\mathcal{L}$ to be the propositional language generated from $\mathcal{P}$, using the standard Boolean connectives $\wedge$ (conjunction), $\vee$ (disjunction), $\rightarrow$ (implication), $\leftrightarrow$ (equivalence), $\neg$ (negation), the special symbol $\perp$ (arbitrary contradiction), and governed by classical propositional logic. In particular, the logic is identified by its consequence operation $C n$. The operation $C n$, which is a function mapping sets of sentences to sets of sentences, is assumed to satisfy the following properties, for all sets of sentences $\Gamma, \Gamma^{\prime}$, and all sentences $\varphi, \psi$ of $\mathcal{L}$ :

2. Axiom (P), as well as conditions (P1) and (P2), will be discussed in Section 4. 

Inclusion:
$\Gamma \subseteq C n(\Gamma)$.
Iteration:
$C n(\Gamma)=C n(C n(\Gamma))$.
Monotonicity:
If $\Gamma \subseteq \Gamma^{\prime}$, then $C n(\Gamma) \subseteq C n\left(\Gamma^{\prime}\right)$.
Supra-classicality:
$\varphi \in C n(\Gamma)$, if $\Gamma$ classically implies $\varphi$.
Deduction:
$\psi \in C n(\Gamma \cup\{\varphi\})$ iff $(\varphi \rightarrow \psi) \in C n(\Gamma)$.
Compactness:
If $\varphi \in C n(\Gamma)$, then $\varphi \in C n\left(\Gamma^{\prime}\right)$ for some finite $\Gamma^{\prime} \subseteq \Gamma$.

Intuitively speaking, $C n(\Gamma)$ is the set of all logical consequences of $\Gamma$. The inference relation $\models$ is used in the following sense:

$$
\Gamma \models \varphi \quad \text { iff } \quad \varphi \in C n(\Gamma)
$$

In a similar vein, we also sometimes write $\Gamma \models \Gamma^{\prime}$ as $\Gamma^{\prime} \subseteq C n(\Gamma), \psi \models \varphi$ as $\varphi \in C n(\psi)$, and $\models \varphi$ as an alternative notation for $\varphi \in C n(\varnothing)$.

A sentence $\varphi$ of $\mathcal{L}$ is contingent iff $\not \models \varphi$ and $\not \models \neg \varphi$. For a set of sentences (or literals) $\Gamma$ of $\mathcal{L}$, we denote by $\bar{\Gamma}$ the set of negated elements of $\Gamma$; i.e., $\bar{\Gamma}=\{\neg \varphi: \varphi \in \Gamma\}$. We shall write $C n\left(\varphi_{1}, \ldots, \varphi_{n}\right)$ for sentences $\varphi_{1}, \ldots, \varphi_{n}$, as an abbreviation of $C n\left(\left\{\varphi_{1}, \ldots, \varphi_{n}\right\}\right)$. For any two sentences $\varphi, \psi$ of $\mathcal{L}$, we shall write $\varphi \equiv \psi$ iff $C n(\varphi)=C n(\psi)$.

An agent's set of beliefs will be modelled by a theory, also referred to as a belief set. A theory $K$ of $\mathcal{L}$ is any set of sentences of $\mathcal{L}$ closed under $C n$; in symbols, $K=C n(K)$. We denote the set of all consistent theories of $\mathcal{L}$ by $\mathbb{K}$. For a theory $K$ and a set of sentences $\Gamma$ of $\mathcal{L}$, we denote by $K+\Gamma$ the closure under $C n$ of $K \cup \Gamma$; in symbols, $K+\Gamma=C n(K \cup \Gamma)$. For a sentence $\varphi$ of $\mathcal{L}$, we shall write $K+\varphi$ as an abbreviation of $K+\{\varphi\}$.

A literal is a propositional variable $p \in \mathcal{P}$ or its negation. For a set of literals $Q$, by $\bigvee Q$ we denote the disjunction of the literals in $Q$. We will, sometimes, represent the negation of a propositional variable $p$ as $\bar{p}$, for the sake of readability.

We define a possible world (or simply a world) $r$ to be a consistent set of literals, such that for any propositional variable $p \in \mathcal{P}$, either $p \in r$ or $\neg p \in r$. The set of all possible worlds is denoted by $\mathbb{M}$. For a set of sentences $\Gamma$ of $\mathcal{L},[\Gamma]$ denotes the set of all possible worlds that satisfy $\Gamma$; i.e., $[\Gamma]=\{r \in \mathbb{M}: r \models \Gamma\}$. We use the notation $[\varphi]$, for a sentence $\varphi \in \mathcal{L}$, as an abbreviation of $[\{\varphi\}]$. For a set of worlds $V \subseteq \mathbb{M}$, we denote by $\operatorname{th}(V)$ the set of sentences of $\mathcal{L}$ satisfied by all worlds in $V$; i.e., $\operatorname{th}(V)=\{\varphi \in \mathcal{L}: r \mid=\varphi$, for all $r \in V\}$. If $V$ is empty, then we define $t h(V)=\mathcal{L}$. Obviously, the set $t h(V)$ is always a theory of $\mathcal{L}$. We will, often, identify a possible world with the sequence (rather than set) of literals it satisfies.

Furthermore, some definitions on preorders. A preorder over a set $V$ is any reflexive, transitive binary relation in $V$. The preorder $\preceq$ is total iff, for all $r, r^{\prime} \in V, r \preceq r^{\prime}$ or $r^{\prime} \preceq r$. In addition, for any $X \subseteq V, \min (X, \preceq)$ denotes the set of minimal elements in $X$, with respect to $\preceq$; i.e., $\min (X, \preceq)=\left\{r \in X\right.$ : for all $r^{\prime} \in X$, if $r^{\prime} \preceq r$, then $\left.r \preceq r^{\prime}\right\}$.

We often consider sublanguages of $\mathcal{L}$. Let $Q$ be a (strict) subset of the set of propositional variables $\mathcal{P}$. We denote by $\mathcal{L}^{Q}$ the sublanguage of $\mathcal{L}$ defined over $Q$. In the limiting case where $Q$ is empty, we take $\mathcal{L}^{Q}$ to be the language generated by $\perp$, and the Boolean connectives. For a sentence $x$ of $\mathcal{L}$, we denote by $\mathcal{L}_{x}$ the minimal language of $\mathcal{L}$, within 
which $x$ can be expressed. ${ }^{3}$ If $x$ is inconsistent or a tautology, we take $\mathcal{L}_{x}$ to be $\mathcal{L}^{\varnothing} .4$ Moreover, by $\mathcal{P}_{x}$ we denote the propositional variables in the minimal language of $x$, and by $\overline{\mathcal{L}_{x}}$ the language $\mathcal{L}^{\mathcal{P}-\mathcal{P}_{x}}$. Lastly, for a non-empty set of sentences $\Gamma$ of $\mathcal{L}, \mathcal{L}_{\Gamma}$ denotes the minimal language of $\mathcal{L}$ within which all the sentences of $\Gamma$ can be expressed.

Finally, we shall sometimes project operations defined above for the entire language $\mathcal{L}$, to one of its sublanguages $\mathcal{L}^{\prime}$. When this is the case, all notation will be subscripted by the sublanguage $\mathcal{L}^{\prime}$. For instance, $C n_{\mathcal{L}^{\prime}}(\Gamma)$ denotes the logical closure of a set of sentences $\Gamma$ in $\mathcal{L}^{\prime}$, i.e., $C n_{\mathcal{L}^{\prime}}(\Gamma)=C n(\Gamma) \cap \mathcal{L}^{\prime}$, and $V_{\mathcal{L}^{\prime}}$ denotes the restriction of a set $V$ of possible worlds to $\mathcal{L}^{\prime}$, i.e., $V_{\mathcal{L}^{\prime}}=\left\{r \cap \mathcal{L}^{\prime}\right.$ : for all $\left.r \in V\right\}$. It is clear that the operation is relative to the original language $\mathcal{L}$ when no subscript is present.

\section{The AGM Paradigm}

In this section, the axiomatic approach of the AGM paradigm is briefly discussed, along with three well-known explicit constructions for the process of belief revision. The first construction is based on preorders over possible worlds (faithful-preorders model), the second on preorders over sentences (epistemic-entrenchment model), and the third on preorders over sets of sentences/remainders (partial-meet model).

\subsection{The AGM Postulates for Revision}

In the AGM paradigm, belief revision is modelled as a (binary) function $*$, mapping a theory $K$ and a sentence $\varphi$ to the theory $K * \varphi$, representing the result of revising $K$ by $\varphi$; i.e., $*: \mathbb{K} \times \mathcal{L} \mapsto \mathbb{K}$. The AGM postulates for revision $(K * 1)-(K * 8)$, listed below, appear to capture much of what characterizes rational belief revision. ${ }^{5}$

$$
\begin{array}{ll}
(\mathbf{K} * \mathbf{1}) & K * \varphi \text { is a theory of } \mathcal{L} . \\
(\mathbf{K} * \mathbf{2}) & \varphi \in K * \varphi \\
(\mathbf{K} * \mathbf{3}) & K * \varphi \subseteq K+\varphi \\
(\mathbf{K} * \mathbf{4}) & \text { If } \neg \varphi \notin K, \text { then } K+\varphi \subseteq K * \varphi . \\
(\mathbf{K} * \mathbf{5}) & K * \varphi \mid=\perp \text { iff } \models \neg \varphi . \\
(\mathbf{K} * \mathbf{6}) & \text { If } \varphi \equiv \psi, \text { then } K * \varphi=K * \psi \\
(\mathbf{K} * \mathbf{7}) & K *(\varphi \wedge \psi) \subseteq(K * \varphi)+\psi \\
(\mathbf{K} * \mathbf{8}) & \text { If } \neg \psi \notin K * \varphi, \text { then }(K * \varphi)+\psi \subseteq K *(\varphi \wedge \psi) .
\end{array}
$$

The functions satisfying the above postulates are known as AGM revision functions (or, simply, revision functions). A guiding intuition in formulating $(K * 1)-(K * 8)$ has been

3. That is to say, $\mathcal{L}_{x}$ contains a sentence that is logically equivalent to $x$, and, moreover, no proper sublanguage of $\mathcal{L}_{x}$ contains such a sentence.

4. For every sentence $x$ of $\mathcal{L}, \mathcal{L}_{x}$ is unique (Parikh, 1999).

5. Gärdenfors (1988) and Peppas (2008) present an extended discussion on the postulates. 
the principle of minimal change, according to which a rational agent ought to change her beliefs as little as possible, in order to (consistently) accommodate the new information.

The AGM postulates for revision do not suffice to uniquely determine the belief set $K * \varphi$ resulting from revising $K$ by $\varphi$, given $K$ and $\varphi$ alone; they simply intend to circumscribe the territory of all different rational ways of revising belief sets. What is necessary apart from the above axiomatic approach, are constructive models for the process of belief revision. In the rest of this section, the most well-known models shall be discussed.

Note that, for ease of presentation, in what follows, we shall consider only consistent belief sets, and contingent epistemic input.

\subsection{Faithful-Preorders Model}

One popular construction, introduced by Katsuno and Mendelzon (1991), is based on total preorders over possible worlds, called faithful preorders. ${ }^{6}$

Definition 1 (Faithful Preorder, Katsuno \& Mendelzon, 1991). For a theory $K$ of $\mathcal{L}$, a preorder over possible worlds $\preceq_{K}$ is said to be faithful to $K$ iff it is total, and such that the minimal worlds (with respect to $\preceq_{K}$ ) are those satisfying $K$; i.e., $\min \left(\mathbb{M}, \preceq_{K}\right)=[K] .{ }^{7}$

Given a faithful preorder $\preceq_{K}$ for every theory $K \in \mathbb{K}$, one can construct a revision function $*$, by means of the following condition (Katsuno \& Mendelzon, 1991):

$$
(\mathbf{F} *) \quad K * \varphi=\operatorname{th}\left(\min \left([\varphi], \preceq_{K}\right)\right) .
$$

Intuitively, $\preceq_{K}$ represents a plausibility ranking over possible worlds; the more plausible a world $r$ is, the lower it appears in the ranking. Hence, $(\mathrm{F} *)$ essentially defines $K * \varphi$ as the theory corresponding to the most plausible worlds, satisfying the new information $\varphi$; i.e., the $\preceq_{K}$-minimal $\varphi$-worlds.

Katsuno and Mendelzon have shown that the functions induced from faithful preorders via $(\mathrm{F} *)$ are precisely those satisfying the AGM postulates for revision.

\subsection{Epistemic-Entrenchment Model}

The second constructive model that is discussed herein has been proposed by Gärdenfors and Makinson (1988), and it is based on the notion of epistemic entrenchment. An epistemic entrenchment assigns an epistemic value to the agent's individual beliefs, which in turn determines their fate during revision.

Definition 2 (Epistemic-Entrenchment Ordering, Gärdenfors \& Makinson, 1988). An ordering $\leq_{K}$ of epistemic entrenchment, related to a theory $K$ of $\mathcal{L}$, is a binary relation in $\mathcal{L}$ satisfying the following postulates: ${ }^{8}$

6. The constructive model of Katsuno and Mendelzon is, essentially, a subsequent reformulation of system of spheres, introduced by Grove (1988).

7. To be precise, Katsuno and Mendelzon associate faithful preorders with sentences, rather than belief sets. However, the two approaches are equivalent, given that we are working with a finitary propositional language.

8. Gärdenfors (1988) and Peppas (2008) present an extended discussion on the postulates. 
(EE1) For all $\varphi, \psi, \chi \in \mathcal{L}$, if $\varphi \leq_{K} \psi$ and $\psi \leq_{K} \chi$, then $\varphi \leq_{K} \chi$.

(EE2) For all $\varphi, \psi \in \mathcal{L}$, if $\varphi=\psi$, then $\varphi \leq_{K} \psi$.

(EE3) For all $\varphi, \psi \in K, \varphi \leq_{K} \varphi \wedge \psi$ or $\psi \leq_{K} \varphi \wedge \psi$.

(EE4) When $K \neq \mathcal{L}, \varphi \notin K$ iff $\varphi \leq_{K} \psi$, for all $\psi \in \mathcal{L}$.

(EE5) If $\psi \leq_{K} \varphi$ for all $\psi \in \mathcal{L}$, then $\models \varphi$.

From the above postulates, it follows that an ordering of epistemic entrenchment is a total preorder in $\mathcal{L}$.

Intuitively, $\leq_{K}$ represents the relative epistemic loss caused by the removal of a belief from $K$; the higher a belief is in the epistemic-entrenchment preorder $\leq_{K}$, the more is lost in terms of epistemic value by its removal from $K$. Consequently, for any two sentences $\varphi$ and $\psi$, such that $\varphi \leq_{K} \psi$, whenever a choice exists between giving up $\varphi$ and giving up $\psi$, the former will be surrendered in order to minimize the epistemic loss.

Gärdenfors and Makinson (1988) proved that the revision functions induced from epistemic-entrenchment preorders, by means of (E*) below, are precisely those satisfying the AGM postulates for revision (where $\leq_{K}$ is an epistemic-entrenchment preorder related to every theory $K \in \mathbb{K}):^{9}$

$$
\text { (E*) } \quad \psi \in K * \varphi \quad \text { iff } \quad \text { either } \neg \varphi<_{K} \neg \varphi \vee \psi \quad \text { or } \quad \models \neg \varphi \text {. }
$$

\subsection{Partial-Meet Model}

Before presenting the partial-meet model introduced by Alchourrón et al. (1985), some definitions are in order.

Definition 3 (Remainder Set, Alchourrón et al., 1985). For a theory $K$ of $\mathcal{L}$, and a sentence $\varphi$ such that $\varphi \in K$, a $\varphi$-remainder of $K$ is any maximal subset of $K$ that fails to entail $\varphi$. Formally, a $\varphi$-remainder of $K$ is a subset $K^{\prime}$ of $K$, such that $K^{\prime} \not \models \varphi$, and for any $K^{\prime \prime} \subseteq K$, if $K^{\prime} \subset K^{\prime \prime}$ then $K^{\prime \prime}=\varphi$.

We shall denote the set of all $\varphi$-remainders of $K$ by $K \perp \varphi$.

Definition 4 (Selection Function, Alchourrón et al., 1985). For every theory $K$ of $\mathcal{L}$, a selection function is any function $\gamma$ that maps a non-empty collection $X$ of subsets of $K$ to a non-empty subset $\gamma(X)$ of $X$; i.e., $\varnothing \neq \gamma(X) \subseteq X$.

Intuitively, a selection function picks up the epistemically most entrenched $\varphi$-remainders of $K$, for every $K \in \mathbb{K}$.

The selection function $\gamma$ is transitively relational iff it can be produced from a transitive binary relation $\leqq_{K}$ in $2^{K}$, by means of the following condition:

$$
\gamma(K \perp \varphi)=\left\{K^{\prime} \in K \perp \varphi: K^{\prime \prime} \leqq_{K} K^{\prime} \text { for all } K^{\prime \prime} \in K \perp \varphi\right\} .
$$

9. To be precise, Gärdenfors and Makinson established a connection between epistemic-entrenchment preorders and contraction functions, denoted by - . However, in view of the Levi identity (i.e., $K * \varphi=(K \dot{\neg} \varphi)+\varphi)$, the connection with revision functions, mentioned above, follows directly. Note that condition (E*) appears in the work of Lindström and Rabinowicz (1991), and Rott (1991a). 
It turns out that the ordering $\leqq_{K}$, as defined above, is a total preorder in $K \perp \varphi$ (Gärdenfors, 1988). In what follows, we shall refer to $\leqq_{K}$ as selection-function preorder.

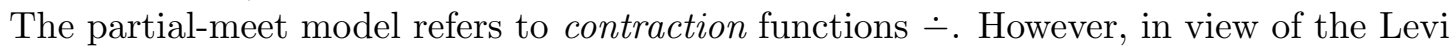
identity, that is, $K * \varphi=(K \dot{-} \neg \varphi)+\varphi$, we get the (partial-meet) revision of $K$ by any sentence $\varphi$, by means of the following condition:

$$
(\mathbf{P M} *) \quad K * \varphi=(\bigcap \gamma(K \perp \neg \varphi))+\varphi .
$$

When $\gamma$ is transitively relational, the revision functions induced from $(\mathrm{PM} *)$ satisfy the AGM postulates for revision.

\subsection{Interrelations}

Given the connection between faithful preorders and revision functions, which in turn are connected to epistemic-entrenchment preorders, it should in principle be possible to establish a direct connection between faithful preorders and epistemic-entrenchment preorders, for every theory $K$ of $\mathcal{L}$. Indeed, this connection is expressed by the following condition (Peppas \& Williams, 1995):

(EF) For any two contingent sentences $\varphi, \psi \in \mathcal{L}, \varphi \leq_{K} \psi$ iff, for some $r \in[\neg \varphi], r \preceq K r^{\prime}$, for every $r^{\prime} \in[\neg \psi]$.

Theorem 1 follows almost immediately and connects revision functions to epistemicentrenchment preorders and faithful preorders.

Theorem 1 (Peppas \& Williams, 1995). Let $K$ be a theory of $\mathcal{L}$. Moreover, let $\leq_{K}$ be an epistemic-entrenchment preorder, and let $\preceq_{K}$ be a faithful preorder. Then, $\leq_{K}$ and $\preceq_{K}$ correspond to the same revision function at $K$, by means of (E*) and ( $\left.F_{*}\right)$ respectively, iff they satisfy condition (EF).

In a similar vein, condition (EP), appearing slightly different in (Rott, 1991b), captures the relationship between epistemic-entrenchment and selection-function preorders:

(EP) For any two contingent sentences $\varphi, \psi \in \mathcal{L}, \varphi \leq_{K} \psi$ iff, for some $\varphi$-remainder $R$ of $K, R^{\prime} \leqq_{K} R$, for every $\psi$-remainder $R^{\prime}$ of $K$.

Theorem 2 connects revision functions to epistemic-entrenchment and selection-function preorders.

Theorem 2 (Rott, 1991b). Let $K$ be a theory of $\mathcal{L}$. Moreover, let $\leq_{K}$ be an epistemicentrenchment preorder, and let $\leqq_{K}$ be a selection-function preorder. Then, $\leq_{K}$ and $\leqq_{K}$ correspond to the same revision function at $K$, by means of ( $\left.E_{*}\right)$ and (PM*) respectively, iff they satisfy condition (EP).

\section{Relevance in Belief Revision}

When revising a theory $K$ by a sentence $\varphi$, it seems plausible to assume that only the beliefs that are relevant to $\varphi$ should be affected, while the rest of the belief corpus should 
remain unchanged. For instance, an agent that is revising her beliefs about the monetary policy of Greece, is unlikely to revise her beliefs about quantum electrodynamics. This simple intuition is not fully captured by the AGM paradigm. To see this, the following two examples should be taken into consideration.

Example 1. Assume that $\mathcal{P}=\{a, b\}$, and let $K$ be the theory $K=C n(a, b)$. There exists an AGM revision function $*$, such that $K *(\neg a)=C n(\neg a, \neg b)$. That is to say, the revision of $K$ by $\neg a$ (modulo $*$ ) results in believing $\neg b$, even if $b$ is irrelevant to $a$, with respect to the belief set $K$.

Example 2 (Trivial AGM Revision Function, Parikh, 1999). Consider the following revision policy, encoded by the (trivial) revision function $*_{\text {trivial }}$, for any theory $K$ and any sentence $\varphi$ of $\mathcal{L}$ :

$$
K *_{\text {trivial }} \varphi= \begin{cases}K+\varphi, & \text { if } \varphi \text { is consistent with } K \\ C n(\varphi), & \text { otherwise }\end{cases}
$$

It turns out that $*_{\text {trivial }}$ satisfies $(K * 1)-(K * 8)$, yet, it is unsatisfactory since, in case $\varphi$ contradicts $K$, all beliefs in $K$ that are not logical consequences of $\varphi$ are thrown away.

Accordingly, Parikh (1999) proposed the new axiom (P), presented below, to supplement the AGM postulates for revision. The main intuition that axiom $(\mathrm{P})$ aims to capture is that, if an agent's beliefs can be subdivided into disjoint compartments referring to different subject matters, then, when revising, the agent modifies only the compartment(s) affected by the new information. Although axiom (P) may not tell the whole story of relevance-sensitive belief revision (it only deals with a special case), it is surely an intuitive first step.

(P) If $K=C n(x, y)$, where $x, y$ are sentences of disjoint sublanguages $\mathcal{L}_{x}, \mathcal{L}_{y}$, respectively, and $\varphi \in \mathcal{L}_{x}$, then $K * \varphi=\left(C n_{\mathcal{L}_{x}}(x) \diamond \varphi\right)+y$, where $\diamond$ is a revision operator of the sublanguage $\mathcal{L}_{x}$.

Parikh (1999) showed that axiom $(\mathrm{P})$ is consistent with postulates $(K * 1)-(K * 6)$ (known as the basic AGM postulates for revision). In a further analysis conducted by Peppas et al. (2015), it was shown that $(\mathrm{P})$ is in fact consistent with the full set of AGM postulates for revision. In this latter work, two different interpretations of $(\mathrm{P})$ were identified, called the weak and the strong version of $(\mathrm{P})$, both of which are plausible depending on the context.

According to the first reading, i.e., the weak version of axiom $(\mathrm{P})$, the revision function $\diamond$ that modifies the relevant part of $K$ - call it the local revision function - may vary from theory to theory, even when the relevant part $C n(x)$ stays the same. This means that weak (P) allows the local revision function to be context-dependent.

For example, consider a revision function $*$ (which defines a revision policy for all theories of $\mathcal{L})$, and let $K=C n(x, y)$ and $H=C n(x, z)$ be two theories of $\mathcal{L}$, such that $\mathcal{L}_{x} \cap \mathcal{L}_{y}=$ $\mathcal{L}_{x} \cap \mathcal{L}_{z}=\varnothing$, and $y \not \equiv z$. Consider, now, any sentence $\varphi \in \mathcal{L}_{x}$. The $\varphi$-relevant part of $K$ and $H$ is, in both cases, the same; this is not the case for the $\varphi$-irrelevant part. However, 


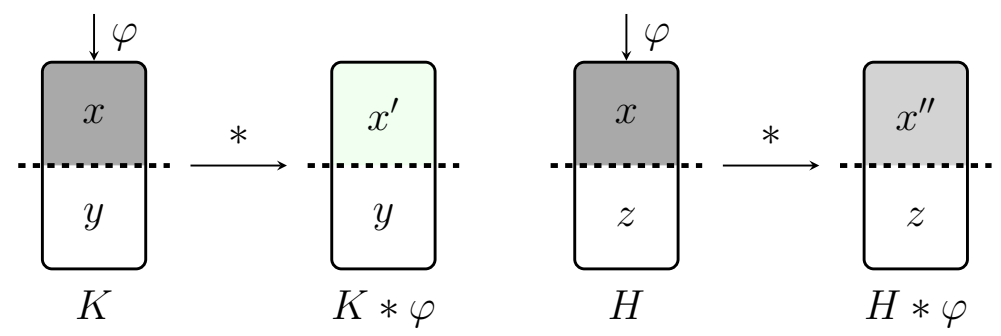

Figure 1: The weak version of axiom (P)

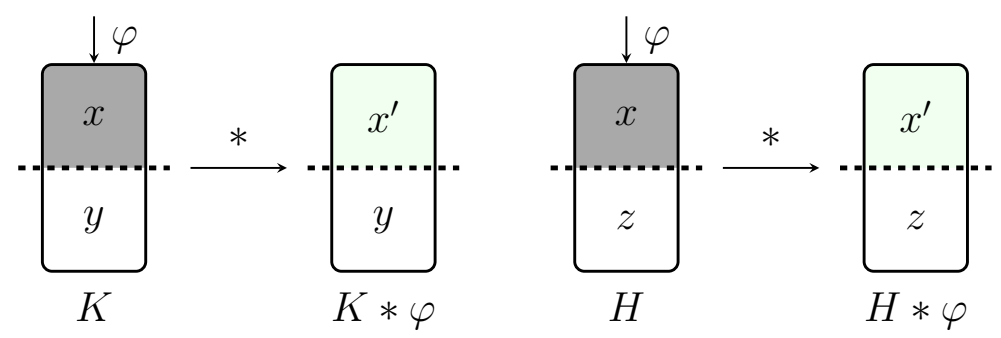

Figure 2: The strong version of axiom $(\mathrm{P})$

weak $(\mathrm{P})$ allows this relevant part to be modified in a different way, for theories $K$ and $H$. In other words, while the non-relevant part to $\varphi$ remains unaffected during the (global) revision by $\varphi$, its presence can influence the way that the $\varphi$-relevant part of the theory is modified (Figure 1).

According to the second reading, i.e., the strong version of axiom $(\mathrm{P})$, the local revision function $\diamond$ becomes context-independent. In the previous example, the way the $\varphi$-relevant part is modified in both $K$ and $H$ is the same (Figure 2). ${ }^{10}$

To avoid ambiguity between the two versions, Peppas et al. (2015) reformulated axiom $(\mathrm{P})$ in terms of the following two conditions that do not refer to a local revision operator:

(P1) If $K=C n(x, y), \mathcal{L}_{x} \cap \mathcal{L}_{y}=\varnothing$, and $\varphi \in \mathcal{L}_{x}$, then $(K * \varphi) \cap \overline{\mathcal{L}_{x}}=K \cap \overline{\mathcal{L}_{x}}$.

(P2) If $K=C n(x, y), \mathcal{L}_{x} \cap \mathcal{L}_{y}=\varnothing$, and $\varphi \in \mathcal{L}_{x}$, then $(K * \varphi) \cap \mathcal{L}_{x}=(C n(x) * \varphi) \cap \mathcal{L}_{x}$.

Condition (P1) corresponds to the weak version of axiom $(\mathrm{P})$, and says that, when revising a theory $K$ by a sentence $\varphi$, the part of $K$ that is irrelevant to $\varphi$ is not affected by the revision. To get the strong version of $(\mathrm{P})$, we need to add condition (P2); that is, strong $(\mathrm{P})$ is equivalent to $(\mathrm{P} 1)$ and $(\mathrm{P} 2)$.

Peppas et al. (2015) characterized both versions of axiom (P) in terms of faithful preorders. To make this article self-contained, the aforementioned characterization is presented in the next two sections.

10. Refer to (Peppas et al., 2015) for a more detailed discussion on this issue. 


\section{Faithful-Preorders Characterization of (P1)}

In this section, the faithful-preorders characterization of condition (P1) is presented.

\subsection{Initial Considerations}

First, we introduce some terminology and notation.

Definition 5 (Possible Worlds Difference). The difference between two possible worlds $w, r$ of $\mathbb{M}$, denoted by Diff $(w, r)$, is the set of propositional variables, over which the two worlds disagree; i.e., the symmetric difference of $w$ and $r$. In symbols:

$$
\operatorname{Diff}(w, r)=\{q \in \mathcal{P}: w \models q \text { and } r \models \neg q\} \cup\{q \in \mathcal{P}: w \models \neg q \text { and } r \models q\} .
$$

Example 3. Suppose that $\mathcal{P}=\{a, b, c\}$. Let $w, r$ be the worlds $w=\{a, b, c\}$ and $r=$ $\{\neg a, b, \neg c\}$. Then, Diff $(w, r)=\{a, c\}$.

Definition 6 (Theory Splitting/Splittable Theory, Parikh, 1999). Let $K$ be a theory of $\mathcal{L}$, and let $Q=\left\{Q_{1}, \ldots, Q_{n}\right\}$ be a partition of $\mathcal{P}$; i.e., $\cup Q=\mathcal{P}, Q_{i} \neq \varnothing$, and $Q_{i} \cap Q_{j}=\varnothing$, for all $1 \leqslant i \neq j \leqslant n$. The set $Q$ is a $K$-splitting iff there exist sentences $x_{1} \in \mathcal{L}^{Q_{1}}, \ldots, x_{n} \in$ $\mathcal{L}^{Q_{n}}$, such that $K=C n\left(x_{1}, \ldots, x_{n}\right)$.

Definition 7 (Confined Theory, Parikh, 1999). Let $\mathcal{L}^{\prime}$ be a sublanguage of $\mathcal{L}$. A theory $K$ is confined to $\mathcal{L}^{\prime}$ iff $K=C n\left(K \cap \mathcal{L}^{\prime}\right)$.

As proved by Parikh (1999), for every theory $K$ of $\mathcal{L}$, there is a unique finest $K$-splitting, denoted by $\mathcal{F}$; i.e., one which refines every other $K$-splitting. ${ }^{11}$ That is to say, there is a unique way to consider theory $K$ as being composed of disjoint ("refined") compartments, referring to irrelevant subject matters.

In case a theory $K$ is confined to a sublanguage $\mathcal{L}^{\prime}$ of $\mathcal{L}$, it also "splits" between $\mathcal{L}^{\prime}$ and $\overline{\mathcal{L}^{\prime}}$, with the $\overline{\mathcal{L}^{\prime}}$ part being trivial. Of course, $K$ knows nothing about $\overline{\mathcal{L}^{\prime}}$; i.e., there is no sentence $\xi \in \overline{\mathcal{L}^{\prime}}$, such that $K \models \xi$. Notice, lastly, that, whenever $n=1$ in Definition $6, K$ is a trivially splittable theory of $\mathcal{L}$.

Example 4. Suppose that $\mathcal{P}=\{a, b, c, d, e, f\}$, and let $K=C n(a \rightarrow(b \vee c), d \leftrightarrow e)$. It is not hard to verify that the finest $K$-splitting is $\mathcal{F}=\{\{a, b, c\},\{d, e\},\{f\}\}$. The set $Q=\{\{a, b, c, d, e\},\{f\}\}$ is a $K$-splitting as well, but not the finest. Observe that theory $K$ has no information about propositional variable $f$, since $K$ is confined to the sublanguage $\mathcal{L}^{\{a, b, c, d, e\}}$.

Definition 8, below, extends Definition 5, in order to include the difference between a theory $K$ of $\mathcal{L}$ and a possible world $r$ of $\mathbb{M}$.

Definition 8 (Difference Between Theories and Possible Worlds, Peppas et al., 2015). Let $K$ be a theory of $\mathcal{L}$, and let $\mathcal{F}=\left\{F_{1}, \ldots, F_{n}\right\}$ be the finest $K$-splitting. Moreover, let $r$ be $a$ possible world of $\mathbb{M}$. The difference between $K$ and $r$, denoted by Diff $(K, r)$, is the union of the elements $F_{i}$ of $\mathcal{F}$, for which there exists a sentence $\varphi$ that can be expressed in the sublanguage $\mathcal{L}^{F_{i}}$, on that $K$ and $r$ disagree. In symbols:

11. A partition $Q^{\prime}$ refines another partition $Q$ iff every element of $Q^{\prime}$ is a subset of some element of $Q$. Equivalently, a partition $Q^{\prime}$ refines another partition $Q$ iff, for every $Q_{i}^{\prime} \in Q^{\prime}$, there is $Q_{j} \in Q$, such that $Q_{i}^{\prime} \subseteq Q_{j}$. 


$$
\operatorname{Diff}(K, r)=\bigcup\left\{F_{i} \in \mathcal{F}: \text { for some } \varphi \in \mathcal{L}^{F_{i}}, K \models \varphi \text { and } r \models \neg \varphi\right\} \text {. }
$$

Example 5. Let $\mathcal{P}=\{a, b, c, d\}$, and $K=C n(a \leftrightarrow b, c \leftrightarrow d)$. The finest $K$-splitting is $\mathcal{F}=\{\{a, b\},\{c, d\}\}$. Now, let $r$ be the world $r=\{\neg a, b, c, d\}$. Then, Diff $(K, r)=\{a, b\}$, since $K$ and $r$ disagree on at least one sentence $\varphi$ that can be expressed in $\mathcal{L}^{\{a, b\}}$ (e.g., for $\varphi=a \vee \neg b, K \mid=\varphi$ and $r \mid=\neg \varphi)$.

Clearly, in the special case of a complete theory $K$ (where there is a world $w$, such that $[K]=\{w\}$ ), the extended definition of Diff collapses to the one given for the difference between two possible worlds.

\subsection{Characterization Conditions}

Having defined the extended Diff, the appropriate faithful-preorders characterization of (P1) turns out to be conditions (Q1) and (Q2) (for a theory $K$ of $\mathcal{L}):{ }^{12}$

(Q1) If Diff $(K, r) \subset \operatorname{Diff}\left(K, r^{\prime}\right)$ and $\operatorname{Diff}\left(r, r^{\prime}\right) \cap \operatorname{Diff}(K, r)=\varnothing$, then $r \prec_{K} r^{\prime}$.

(Q2) If $\operatorname{Diff}(K, r)=\operatorname{Diff}\left(K, r^{\prime}\right)$ and $\operatorname{Diff}\left(r, r^{\prime}\right) \cap \operatorname{Diff}(K, r)=\varnothing$, then $r \approx_{K} r^{\prime}$.

\subsection{Characterization Result}

Theorem 3 shows that conditions (Q1)-(Q2) characterize precisely the family of faithful preorders corresponding to AGM revision functions satisfying (P1).

Theorem 3 (Peppas et al., 2015). Let $*$ be a revision function that satisfies $(K * 1)-(K * 8)$, and $\left\{\preceq_{K}\right\}_{K \in \mathbb{K}}$ a family of faithful preorders (one for each theory $K$ of $\mathcal{L}$ ), corresponding to * by means of (F*). Then, * satisfies (P1) iff $\left\{\preceq_{K}\right\}_{K \in \mathbb{K}}$ satisfies (Q1)-(Q2).

\section{Faithful-Preorders Characterization of (P2)}

In this section, the faithful-preorders characterization of condition (P2) is presented.

\subsection{Initial Considerations}

First, we introduce some more notation. For a contingent sentence $x \in \mathcal{L}$ and a possible world $r \in \mathbb{M}$, by $r_{x}$ is denoted the restriction of $r$ to the minimal language of $x$; i.e., $r_{x}=r \cap \mathcal{L}_{x}$.

Definition 9 (Faithful-Preorder Filtering, Peppas et al., 2015). Let $K$ be a theory of $\mathcal{L}$. For a faithful preorder $\preceq_{K}$ and a contingent sentence $x \in \mathcal{L}$, the $x$-filtering of $\preceq_{K}$, denoted by $\preceq_{K}^{x}$, is as follows:

$$
r \preceq_{K}^{x} r^{\prime} \quad \text { iff } \quad \text { there is a world } w \in\left[r_{x}\right] \text {, such that, for all } w^{\prime} \in\left[r_{x}^{\prime}\right], w \preceq_{K} w^{\prime} \text {. }
$$

Intuitively, the $x$-filtering (of a preorder) can be regarded as a "projection/restriction" of the initial preorder to the minimal language of $x$, treating the propositional variables outside

12. $\prec_{K}$ and $\approx_{K}$ denote the strict and symmetric part of $\preceq_{K}$, respectively. 
$\mathcal{P}_{x}$ as invisible. Notice, moreover, that $\preceq_{K}^{x}$ depends entirely on the minimal language of $x$, in the sense that, if $x, z$ are two sentences such that $\mathcal{L}_{x}=\mathcal{L}_{z}$, then $\preceq_{K}^{x}=\preceq_{K}^{z}$, even if $x \not \equiv z$. Subsequently, a concrete example of $x$-filtering is presented.

Example 6 (Peppas et al., 2015). Suppose that $\mathcal{P}=\{a, b, c\}$. Let $K$ be the theory $K=C n(a \leftrightarrow \neg b, c)$, and $\preceq_{K}$ be the total preorder over the possible worlds of $\mathbb{M}$ shown below:

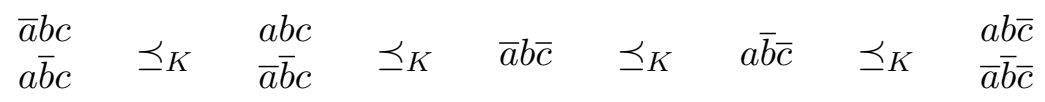

Now, consider the (arbitrary) sentence $x=(a \vee b \vee c) \wedge(a \vee b \vee \neg c)$, for which $\mathcal{L}_{x}=\mathcal{L}^{\{a, b\}}$, since $x$ is logically equivalent to $a \vee b$ and no sentence with fewer propositional variables is logically equivalent to $x$. Then, the $x$-filtering of $\preceq_{K}$ is the following:

$\begin{array}{lll}\bar{a} b c & & a b c \\ \bar{a} b \bar{c} & \preceq_{K}^{x} & a b \bar{c} \\ a \bar{b} c & \bar{a} \bar{b} c \\ a \bar{b} \bar{c} & & \bar{a} \bar{b} \bar{c}\end{array}$

It is not hard to verify that, for any contingent sentence $x \in \mathcal{L}$, if $\preceq_{K}$ is a total preorder, then so is $\preceq_{K}^{x}$. In addition, if $\mathcal{L}_{x}=\mathcal{L}$, then $x$-filtering has no effect on $\preceq_{K}$; i.e., $\preceq_{K}^{x}=\preceq_{K}$.

\subsection{Characterization Condition}

Condition (Q3), below, turns out to be the faithful-preorders characterization of (P2) (the preorders $\preceq_{K}$ and $\preceq_{C n(x)}$ are faithful to $K$ and $C n(x)$, respectively):

(Q3) If $K=C n(x, y)$ and $\mathcal{L}_{x} \cap \mathcal{L}_{y}=\varnothing$, then $\preceq_{K}^{x}=\preceq_{C n(x)}^{x}$.

Condition (Q3), essentially, says that, whenever a belief set $K$ can be split into two disjoint compartments $C n(x)$ and $C n(y)$, then the $x$-filtering of the preorder $\preceq_{K}$, associated with $K$, ought to be identical with the $x$-filtering of the preorder $\preceq_{C n(x)}$, associated with the first compartment of $K$. Obviously, (Q3) makes associations between faithful preorders related to different (overlapping) theories. This is to be expected, since condition (P2) introduces dependencies between revision policies associated with different theories.

\subsection{Characterization Result}

Theorem 4 shows that condition (Q3) characterizes precisely the family of faithful preorders corresponding to AGM revision functions satisfying condition (P2).

Theorem 4 (Peppas et al., 2015). Let $*$ be a revision function that satisfies $(K * 1)-(K * 8)$, and $\left\{\preceq_{K}\right\}_{K \in \mathbb{K}}$ a family of faithful preorders (one for each theory $K$ of $\mathcal{L}$ ), corresponding to * by means of (F*). Then, * satisfies (P2) iff $\left\{\preceq_{K}\right\}_{K \in \mathbb{K}}$ satisfies (Q3). 


\subsection{Combining the Results}

Putting together the results of Theorems 3 and 4, we obtain immediately the following theorem that provides the faithful-preorders characterization of (the strong version of) Parikh's relevance-sensitive axiom.

Theorem 5 (Peppas et al., 2015). Let $*$ be a revision function that satisfies $(K * 1)-(K * 8)$, and $\left\{\preceq_{K}\right\}_{K \in \mathbb{K}}$ a family of faithful preorders (one for each theory $K$ of $\mathcal{L}$ ), corresponding to * by means of $(F *)$. Then, $*$ satisfies strong $(P)$ iff $\left\{\preceq_{K}\right\}_{K \in \mathbb{K}}$ satisfies (Q1)-(Q3).

Before closing this section, it is worth noting that Kern-Isberner and Brewka (2017) generalized the results of Peppas et al. (2015) — and thus of Parikh (1999) — to the revision of belief states (rather than belief sets). ${ }^{13}$ Note that a belief state (also referred to as an epistemic state) is defined as a belief set, coupled with a structure that encodes relative plausibility (usually, a faithful preorder or a system of spheres). As a consequence, a belief state is a "richer" model than a belief set. Kern-Isberner and Brewka (2017), also, extended the approach of Peppas et al. (2015) to ordinal conditional functions (Spohn, 1988).

In the rest of the article, we proceed to our objective, which is to characterize both conditions (P1) and (P2) in terms of epistemic-entrenchment and partial-meet models.

\section{Epistemic-Entrenchment Characterization of (P1)}

The epistemic-entrenchment characterization of condition (P1) is introduced in this section.

\subsection{Initial Considerations}

The necessary terminology and notation are first introduced, taking into account the $K$ splitting concept, which is central to Parikh's notion of relevance.

Definition 10 (Theory Units). Let $K$ be a theory of $\mathcal{L}$, and let $\mathcal{F}=\left\{F_{1}, \ldots, F_{n}\right\}$ be the finest $K$-splitting. Moreover, let $\chi_{1}, \ldots, \chi_{n}$ be sentences of $\mathcal{L}$, such that $\chi_{1} \in \mathcal{L}^{F_{1}}, \ldots, \chi_{n} \in$ $\mathcal{L}^{F_{n}}$, and $K=C n\left(\chi_{1}, \ldots, \chi_{n}\right)$. We shall call sentences $\chi_{1}, \ldots, \chi_{n}$ the units of $K$, and the set $\mathcal{U}=\left\{\chi_{1}, \ldots, \chi_{n}\right\}$ the unit set of $K$.

It can be easily shown that, for every theory $K$ of $\mathcal{L}$, its unit set $\mathcal{U}$ is unique (modulo logical equivalence).

The units of $K$ can be regarded as its "building blocks", since the conjunction of them minimally entails $K$. Moreover, observe that, if $\mathcal{L}_{\mathcal{U}} \subset \mathcal{L}$, then $K$ is confined to the sublanguage $\mathcal{L}_{\mathcal{U}}$. If $\mathcal{L}_{\mathcal{U}}=\mathcal{L}$, theory $K$ is not confined to any sublanguage of $\mathcal{L}$.

In the approach of structured belief bases used for local change by Wassermann (2001b), the unit set $\mathcal{U}$ of $K$ would be a belief base which generates $K$, such that every element of it (unit) constitutes a different connected subgraph. Note that a belief base is a set of sentences that is not (except as a limiting case) closed under logical implication, and for all practical purposes it is in fact finite.

Consider, now, any two contingent sentences $\varphi, \psi$ of $\mathcal{L}$.

13. The extension of relevance-sensitive belief revision to belief states is a necessary prerequisite for covering iterated revision, as captured by Darwiche and Pearl (1997). This is due to the fact that the revision functions defined by Darwiche and Pearl (1997) operate on belief states (rather than on belief sets). 
Definition 11 (Support Set). A support set for $\varphi$ in $K$ is a set $\Gamma$ of units of $K$ that entails $\varphi$; i.e., $\Gamma \subseteq \mathcal{U}$ and $\Gamma \models \varphi$. A support set for $\varphi$ in $K$ is minimal iff no proper subset of it entails $\varphi$. The set of all minimal support sets for $\varphi$ in $K$ is denoted by $S S(\varphi)$.

Intuitively, a minimal support set for $\varphi$ in $K$ is a minimal set of units, that suffices to "justify" the presence of $\varphi$ in $K$.

Definition 12 (Cut). A cut for a sentence $\varphi$ in $K$ is a minimal set of units, whose withdrawal from $K$ will leave $\varphi$ unsupported. Formally, a cut for $\varphi$ in $K$ is a set $\theta$ of units of $K, \theta \subseteq \mathcal{U}$, such that $\mathcal{U}-\theta \not \models \varphi$, and for every proper subset $\theta^{\prime}$ of $\theta, \mathcal{U}-\theta^{\prime}=\varphi$.

Clearly, a cut $\theta$ for a sentence $\varphi$ in $K$ minimally intersects ("cuts") every minimal support set for $\varphi$ in $K$. Thus, it follows that $\theta \subseteq \mathcal{L}_{\cup S S(\varphi)}$.

It should be pointed out that minimal support sets and cuts are closely related to the notions of kernels and incision functions, respectively, of Hansson's approach to contraction of belief bases, called kernel contraction (Hansson, 1994) — we shall discuss this relation later in this article (Subsection 7.4).

Now, we will engage with what we call degree of support of sentences in a theory.

Definition 13 (Degree of Support I). A sentence $\psi$ is better supported than a sentence $\varphi$ in $K$, which we denote by $\varphi \triangleleft_{K} \psi$, iff the following two conditions hold:

(i) For every minimal support set for $\varphi$ in $K$, there exists a minimal support set for $\psi$ in $K$, such that it is a subset of the former; in symbols, $\forall A \in S S(\varphi), \exists B \in S S(\psi)$, such that $B \subseteq A$.

(ii) There exists a minimal support set for $\psi$ in $K$, such that it is disjoint from every minimal support set for $\varphi$ in $K$; in symbols, $\exists C \in S S(\psi)$, such that $C \cap A=\varnothing$, $\forall A \in S S(\varphi)$.

From Definition 13, the following lemma is obviously derived.

Lemma 1. For a theory $K$ and any two sentences $\varphi, \psi$ of $\mathcal{L}, \varphi \triangleleft_{K} \psi$ iff, for every cut $\theta^{\prime}$ for $\psi$ in $K$, there is a cut $\theta$ for $\varphi$ in $K$, such that $\theta \subset \theta^{\prime}$.

Proof. Obvious from the definitions of support set (Definition 11), cut (Definition 12), and Definition 13.

Intuitively, $\psi$ is better supported than $\varphi$ in $K$ iff, whenever one cuts enough "links" to "disconnect" $\psi$ from the units of $K$, regardless of how this is done (there are, in general, more than one ways), $\varphi$ gets "disconnected" as well.

Definition 14 (Degree of Support II). Sentences $\varphi$ and $\psi$ are equally supported in $K$, which we denote by $\varphi \sim_{K} \psi$, iff $\varphi, \psi \in K$, and, moreover, the set of all minimal support sets for $\varphi$ in $K$ is equal to the set of all minimal support sets for $\psi$ in $K$; i.e., $S S(\varphi)=S S(\psi)$.

Analogously, from Definition 14, the following lemma is derived.

Lemma 2. For a theory $K$ and any two sentences $\varphi, \psi$ of $\mathcal{L}, \varphi \sim_{K} \psi$ iff $\varphi, \psi \in K$, and, moreover, the set of all cuts for $\varphi$ in $K$ is equal to the set of all cuts for $\psi$ in $K$. 
Proof. Obvious from the definitions of support set (Definition 11), cut (Definition 12), and Definition 14.

Example 7. Let $\mathcal{P}=\{a, b, c, d, e, f\}$, and $K=C n(a \leftrightarrow b, c \leftrightarrow d, e \leftrightarrow f)$. The finest $K$ splitting is $\mathcal{F}=\{\{a, b\},\{c, d\},\{e, f\}\}$, and the unit set of $K$ is $\mathcal{U}=\{a \leftrightarrow b, c \leftrightarrow d, e \leftrightarrow f\}$.

Let $\varphi=((\neg a \vee b) \wedge(\neg c \vee d)) \vee e \vee f$, and $\psi=a \vee \neg b \vee e \vee \neg f$. Clearly, $\varphi, \psi \in K$. Moreover, $S S(\varphi)=\{\{a \leftrightarrow b, c \leftrightarrow d\}\}$ and $S S(\psi)=\{\{a \leftrightarrow b\},\{e \leftrightarrow f\}\}$. Hence, we derive that $\varphi \triangleleft_{K} \psi$.

Now, let $\varphi=\neg a \vee b \vee c \vee d$, and $\psi=a \vee \neg b \vee \neg c \vee \neg d$. Again, $\varphi, \psi \in K$. In addition, $S S(\varphi)=\{\{a \leftrightarrow b\}\}$ and $S S(\psi)=\{\{a \leftrightarrow b\}\}$. Hence, it follows that $\varphi \sim_{K} \psi$.

The intuitive reading of the above definitions is based on the view that the units of $K$ are the primary beliefs.

The last definition of this subsection refers to sentences of $\mathcal{L}$, making no reference to a theory of the language.

Definition 15 (Logical Equivalence Modulo Sublanguages). For a sublanguage $\mathcal{L}^{\prime}$ of $\mathcal{L}$, two sentences $\varphi$, $\psi$ of $\mathcal{L}$ are logically equivalent modulo $\mathcal{L}^{\prime}$, which is denoted by $\varphi \equiv \mathcal{L}^{\prime} \psi$, iff $[\varphi]_{\mathcal{L}^{\prime}}=[\psi]_{\mathcal{L}^{\prime}} ;$ namely, $\left\{r \cap \mathcal{L}^{\prime}:\right.$ for all $\left.r \in[\varphi]\right\}=\left\{r^{\prime} \cap \mathcal{L}^{\prime}:\right.$ for all $\left.r^{\prime} \in[\psi]\right\}$.

From the above definition, the following lemma is easily derived.

Lemma 3. For a sublanguage $\mathcal{L}^{\prime}$ of $\mathcal{L}$ and two sentences $\varphi, \psi$ of $\mathcal{L}, \varphi \equiv_{\mathcal{L}^{\prime}} \psi$ iff $[\neg \varphi]_{\mathcal{L}^{\prime}}=$ $[\neg \psi]_{\mathcal{L}^{\prime}}$.

Proof. Follows directly from Definition 15 , and the fact that $[\varphi]=\mathbb{M}-[\neg \varphi]$ and $[\psi]=$ $\mathbb{M}-[\neg \psi]$.

Definition 15 implies that, whenever $\mathcal{L}^{\prime}=\mathcal{L}$, it holds that $\varphi \equiv_{\mathcal{L}^{\prime}} \psi$ iff $\varphi \equiv \psi$. In what follows, the sublanguage $\mathcal{L}^{\prime}$ in Definition 15 will be the sublanguage $\mathcal{L}_{\Gamma}$ of some set of sentences $\Gamma$ of $\mathcal{L}$. ${ }^{14}$ When this is the case, we shall use $\varphi \equiv_{\Gamma} \psi$, instead of $\varphi \equiv_{\mathcal{L}^{\prime}} \psi$ or $\varphi \equiv_{\mathcal{L}_{\Gamma}} \psi$.

Example 8. Suppose that $\mathcal{P}=\{a, b, c\}, \varphi=(a \wedge \neg b) \vee c$, and $\psi=(a \wedge \neg b) \vee \neg c$. Consider the sublanguage $\mathcal{L}^{\prime}=\mathcal{L}^{\{a, b\}}$. Then, we derive that $\varphi \equiv_{\mathcal{L}^{\prime}} \psi$. However, for the sentences $\varphi=(a \wedge \neg b) \vee c$ and $\psi=(\neg a \wedge b) \vee \neg c$, it follows that $\varphi \not \equiv \mathcal{L}^{\prime} \psi$.

\subsection{Characterization Conditions}

Having introduced the necessary definitions, we formulate conditions (EP1) and (EP2), below, which turn out to be the epistemic-entrenchment characterization of (P1) (alias, weak $(\mathrm{P})):^{15}$

(EP1) If $\varphi \triangleleft_{K} \psi$ and $\varphi \equiv \cup S S(\varphi) \psi$, then $\varphi<_{K} \psi$.

(EP2) If $\varphi \sim_{K} \psi$ and $\varphi \equiv \cup S S(\varphi) \psi$, then $\varphi \simeq_{K} \psi$.

14. Recall that, for a set of sentences $\Gamma$ of $\mathcal{L}, \mathcal{L}_{\Gamma}$ denotes the minimal sublanguage of $\mathcal{L}$ within which all the sentences of $\Gamma$ can be expressed.

15. $<_{K}$ and $\simeq_{K}$ denote the strict and symmetric part of $\leq_{K}$, respectively. 
To understand the intuition behind conditions (EP1) and (EP2), let us consider a concrete example.

Example 9. Let $\mathcal{P}=\{a, b, c, d\}$, and $K=C n(a \leftrightarrow b, c \leftrightarrow d)$. The finest $K$-splitting is $\mathcal{F}=\{\{a, b\},\{c, d\}\}$, and the unit set of $K$ is $\mathcal{U}=\{a \leftrightarrow b, c \leftrightarrow d\}$.

As for condition (EP1), let $\varphi=a \vee \neg b \vee c \vee d$, and $\psi=a \vee \neg b \vee c \vee \neg d$. Observe that $\varphi, \psi \in K, S S(\varphi)=\{\{a \leftrightarrow b\}\}$, and $S S(\psi)=\{\{a \leftrightarrow b\},\{c \leftrightarrow d\}\}$. Hence, we derive that $\varphi \triangleleft_{K} \psi$. Moreover, $\varphi \equiv \cup S S(\varphi) \psi$, consequently according to (EP1), $\varphi<_{K} \psi$. However, this is not the case for the sentences $\varphi=\neg a \vee b \vee c \vee d$, and $\psi=a \vee \neg b \vee c \vee \neg d$. Here, although $\varphi, \psi \in K, S S(\varphi)=\{\{a \leftrightarrow b\}\}$, and $S S(\psi)=\{\{a \leftrightarrow b\},\{c \leftrightarrow d\}\}$ (thus $\varphi \triangleleft_{K} \psi$ ), it holds that $\varphi \not \equiv \cup S S(\varphi) \psi$. Therefore, the relative order of $\varphi$ and $\psi$, with respect to $\leq_{K}$, is not constrained by (EP1), since its antecedent is not satisfied.

In a similar vein, for condition (EP2), let $\varphi=a \vee \neg b \vee c \vee d$, and $\psi=a \vee \neg b \vee \neg c \vee \neg d$. Observe that $\varphi, \psi \in K, S S(\varphi)=\{\{a \leftrightarrow b\}\}$, and $S S(\psi)=\{\{a \leftrightarrow b\}\}$. Hence, we derive that $\varphi \sim_{K} \psi$. Moreover, $\varphi \equiv \cup S S(\varphi) \psi$, consequently according to (EP2), $\varphi \simeq_{K} \psi$. However, this is not the case for the sentences $\varphi=\neg a \vee b \vee c \vee d$, and $\psi=a \vee \neg b \vee \neg c \vee \neg d$. Here, although $\varphi, \psi \in K, S S(\varphi)=\{\{a \leftrightarrow b\}\}$, and $S S(\psi)=\{\{a \leftrightarrow b\}\}$ (thus $\varphi \sim_{K} \psi$ ), it holds that $\varphi \not \equiv \cup S S(\varphi) \psi$. Therefore, the relative order of $\varphi$ and $\psi$, with respect to $\leq_{K}$, is not constrained by (EP2), since its antecedent is not satisfied.

In the special case of a complete theory as an initial belief set, (EP1) is equivalent to condition (EPC) presented below, while (EP2) reduces to a vacuous condition.

(EPC) If $\varphi \triangleleft_{K} \psi$, then $\varphi<_{K} \psi$.

Condition (EPC) appears in the work of Peppas, Foo, and Nayak (2000) as a constraint on sentences, and has been motivated independently in Belief Revision. Essentially, it associates the epistemic entrenchment of a sentence with the degree of support it has in a theory; the more supported a sentence is in a theory $K$, the higher it appears in the epistemic-entrenchment preorder $\leq_{K}$.

Both conditions (EP1) and (EP2) are variants of (EPC). Indeed, the antecedent of (EP1) is stronger than the one of (EPC), since it requires not only that $\psi$ is better supported than $\varphi$ in $K$, but, moreover, that $\varphi$ and $\psi$ are logically equivalent modulo $\mathcal{L}_{\cup S S(\varphi)}$. Condition (EP2) is, also, in the spirit of (EPC). It deals with the case of two sentences $\varphi$ and $\psi$ that are equally supported in $K$, and, moreover, they are logically equivalent modulo $\mathcal{L}_{\cup S S(\varphi)}$ (where, in this case, it holds that $\mathcal{L}_{\cup S S(\varphi)}=\mathcal{L}_{\cup S S(\psi)}$ ). For such sentences, (EP2) states that they ought to be equally entrenched with respect to $K$.

\subsection{Characterization Result}

Theorem 6 shows that (EP1) and (EP2) characterize precisely the family of epistemicentrenchment preorders corresponding to AGM revision functions satisfying condition (P1).

Theorem 6. Let $*$ be a revision function that satisfies $(K * 1)-(K * 8)$, and let $\left\{\leq_{K}\right\}_{K \in \mathbb{K}}$ be a family of epistemic-entrenchment preorders (one for each theory $K$ of $\mathcal{L}$ ), corresponding to $*$ by means of (E*). Then, $*$ satisfies (P1) iff $\left\{\leq_{K}\right\}_{K \in \mathbb{K}}$ satisfies (EP1)-(EP2). 
Proof. It suffices to establish a connection between conditions (Q1)-(Q2) and conditions (EP1)-(EP2). In particular, we show that, for a faithful preorder $\preceq_{K}$, associated with $*$, and the epistemic-entrenchment preorder $\leq_{K}$, corresponding to $\preceq_{K}$ by means of (EF), $\preceq_{K}$ satisfies (Q1)-(Q2) iff $\leq_{K}$ satisfies $(\mathrm{EP} 1)-(\mathrm{EP} 2)$.

$(\Rightarrow)$

Suppose that $\preceq_{K}$ satisfies (Q1) and (Q2).

For (EP1), assume that, for any two contingent sentences $\varphi, \psi \in \mathcal{L}, \varphi \triangleleft_{K} \psi$ and $\varphi \equiv \cup S S(\varphi) \psi$. We need to show that $\varphi<_{K} \psi$. Notice that from $\varphi \triangleleft_{K} \psi$ it follows that $\psi \in K$. Therefore, if $\varphi \notin K$, (EP1) follows directly from (EE4). Assume, therefore, that $\varphi \in K$.

Consider any world $r^{\prime}$, such that $r^{\prime} \in[\neg \psi]$. Clearly, there is a cut $\theta^{\prime}$ for $\psi$ in $K$, such that $r^{\prime} \models \overline{\theta^{\prime}}{ }^{16}$ Since $\varphi \equiv \cup S S(\varphi) \psi$, it follows from Lemma 3 that $[\neg \varphi]_{\mathcal{L}_{\cup S S(\varphi)}}=[\neg \psi]_{\mathcal{L}_{\cup S S(\varphi)}}$; that is, $\left\{r \cap \mathcal{L}_{\bigcup S S(\varphi)}\right.$ : for all $\left.r \in[\neg \varphi]\right\}=\left\{r^{\prime} \cap \mathcal{L}_{\cup S S(\varphi)}\right.$ : for all $\left.r^{\prime} \in[\neg \psi]\right\}$. Hence, there is a world $r \in[\neg \varphi]$, such that $r$ agrees with $r^{\prime}$ on all propositional variables in $\mathcal{L}_{\cup S S(\varphi)}$, and with a $K$-world on the remaining variables. ${ }^{17}$ Moreover, since $\varphi \triangleleft_{K} \psi$, for every cut $\theta^{\prime}$ for $\psi$ in $K$, there is a cut $\theta$ for $\varphi$ in $K$, such that $\theta \subset \theta^{\prime}$ (with $\mathcal{L}_{\cup S S(\varphi)} \subset \mathcal{L}_{\theta^{\prime}}$ ), from which we derive that $r=\bar{\theta}$. Hence, it follows that $\operatorname{Diff}(K, r) \subset \operatorname{Diff}\left(K, r^{\prime}\right)$ and $\operatorname{Diff}\left(r, r^{\prime}\right)$ $\cap \operatorname{Diff}(K, r)=\varnothing$, therefore from (Q1), it follows that $r \prec_{K} r^{\prime}$. Finally, since for some $r \in[\neg \varphi], r \prec_{K} r^{\prime}$, for every $r^{\prime} \in[\neg \psi]$, we derive from (EF) that $\varphi<_{K} \psi$, as desired.

For (EP2), assume that, for any two contingent sentences $\varphi, \psi \in \mathcal{L}, \varphi \sim_{K} \psi$ and $\varphi \equiv \cup S S(\varphi) \psi$. We need to show that $\varphi \simeq_{K} \psi$. If $\mathcal{L}_{\cup S S(\varphi)}=\mathcal{L}_{\cup S S(\psi)}=\mathcal{L}$, then $\varphi \equiv \psi$, and therefore trivially $\varphi \simeq_{K} \psi$. Assume, therefore, that $\mathcal{L}_{\cup S S(\varphi)}=\mathcal{L}_{\cup S S(\psi)} \subset \mathcal{L}$.

Consider any world $r$, such that $r \in[\neg \varphi]$. Clearly, there is a cut $\theta$ for $\varphi$ in $K$, such that $r \models \bar{\theta}$ (see Footnote 16). Since $\varphi \equiv \cup S S(\varphi) \psi$, it follows from Lemma 3 that $[\neg \varphi]_{\mathcal{L}_{\cup S S(\varphi)}}=$ $[\neg \psi]_{\mathcal{L}_{\cup S S(\varphi)}}$; that is, $\left\{r \cap \mathcal{L}_{\cup S S(\varphi)}\right.$ : for all $\left.r \in[\neg \varphi]\right\}=\left\{r^{\prime} \cap \mathcal{L}_{\cup S S(\varphi)}\right.$ : for all $\left.r^{\prime} \in[\neg \psi]\right\}$. Hence, there is a world $r^{\prime} \in[\neg \psi]$, such that $r^{\prime}$ agrees with $r$ on all propositional variables in $\mathcal{L}_{\bigcup S S(\varphi)}=\mathcal{L}_{\cup S S(\psi)}$, and with a $K$-world on the remaining variables (see Footnote 17). If $r$ agrees with a $K$-world too, on all propositional variables in $\mathcal{L}-\mathcal{L} \cup S S(\varphi)$, then it follows that $\operatorname{Diff}(K, r)=\operatorname{Diff}\left(K, r^{\prime}\right)$ and Diff $\left(r, r^{\prime}\right) \cap \operatorname{Diff}(K, r)=\varnothing$, therefore from (Q2), $r \approx_{K} r^{\prime}$. If $r$ disagrees with all $K$-worlds, on some propositional variables in $\mathcal{L}-\mathcal{L} \cup S S(\varphi)$, then it follows that Diff $\left(K, r^{\prime}\right) \subset \operatorname{Diff}(K, r)$ and Diff $\left(r^{\prime}, r\right) \cap \operatorname{Diff}\left(K, r^{\prime}\right)=\varnothing$, therefore from (Q1), $r^{\prime} \prec_{K} r$. In either case, we derive from (EF) that $\psi \leq_{K} \varphi$. By a totally analogous argument, considering now any world $r^{\prime}$ such that $r^{\prime} \in[\neg \psi]$, we can also prove that $\varphi \leq_{K} \psi$. Consequently, $\varphi \simeq_{K} \psi$ as desired.

16. Assume, on the contrary, that $r^{\prime} \models \theta^{\prime}$, for all cuts $\theta^{\prime}$ for $\psi$ in $K$. Observe that $\left\{\theta^{\prime}: \theta^{\prime}\right.$ is a cut for $\psi$ in $K\}=\bigcup S S(\psi)$. Therefore $r^{\prime} \models \bigcup S S(\psi)$, hence $r^{\prime} \models \psi$. Contradiction.

17. Let $S_{i}$ be the set of worlds in $[\neg \varphi]$, that satisfy the $i$-th element of $R=\left\{z \cap \mathcal{L}_{\cup S S(\varphi)}\right.$ : for all $\left.z \in[\neg \varphi]\right\}$. Clearly, $\bigcup_{i=1}^{|R|} S_{i}=[\neg \varphi]$. It suffices to show that there is a world, in every $S_{i}$, such that it agrees with a $K$-world, on all propositional variables in $\mathcal{L}-\mathcal{L}_{\cup S S(\varphi)}$. Now, assume, for contradiction, that there is no such world in $S_{i}$. Then, for every world $w$ in $S_{i}$, there is a sentence $\nu \in \mathcal{L}-\mathcal{L}_{\cup S S(\varphi)}$, such that $K \models \nu$ and $w \models \neg \nu$. Clearly then, there is a sentence $\zeta \in \mathcal{L}-\mathcal{L}_{\cup S S(\varphi)}$, such that $K \models \zeta$ and $\operatorname{th}\left(S_{i}\right) \models \neg \zeta$. However, $\bigvee_{i=1}^{|R|} \operatorname{th}\left(S_{i}\right) \equiv \neg \varphi$, hence, there is a sentence $\xi \in \mathcal{L}-\mathcal{L} \cup S S(\varphi)$, such that $K=\xi$ and $\neg \varphi \models \neg \xi$ (or $\xi \models \varphi$ ), from which it follows that $K \models \varphi$, through a sentence in $\mathcal{L}-\mathcal{L}_{\cup S S(\varphi)}$. Contradiction. 
$(\Leftarrow)$

Suppose that $\leq_{K}$ satisfies (EP1) and (EP2).

For (Q1), assume that, for any two worlds $r, r^{\prime} \in \mathbb{M}$, Diff $(K, r) \subset$ Diff $\left(K, r^{\prime}\right)$ and $\operatorname{Diff}\left(r, r^{\prime}\right) \cap \operatorname{Diff}(K, r)=\varnothing$. We need to show that $r \prec K r^{\prime}$. If $\operatorname{Diff}(K, r)=\varnothing$, then $r$ is consistent with $K$, and therefore $r \in[K]$ (and of course $r^{\prime} \notin[K]$ ). Then, the faithfulness of $\preceq_{K}$ entails (Q1). Assume, therefore, that Diff $(K, r) \neq \varnothing$.

Construct the sentence $\psi$ as follows: $\psi=\bigvee \overline{r^{\prime}}$. Clearly, $K \models \psi$ and $r^{\prime}=\neg \psi$. ${ }^{18}$ Moreover, by construction, the only cut $\theta^{\prime}$ for $\psi$ in $K$ is the set of all units of $K$, that can be expressed in $\mathcal{L}^{\text {Diff }\left(K, r^{\prime}\right)}$. Now, construct the sentence $\varphi$ as follows: $\varphi=\bigvee \bar{r}$. Clearly, $K \models \varphi$ and $r \models \neg \varphi$. Moreover, by construction, the only cut for $\varphi$ in $K$ is the set of all units of $K$, that can be expressed in $\mathcal{L}^{\text {Diff }(K, r)}$. Since Diff $(K, r) \subset D$ iff $\left(K, r^{\prime}\right)$, it follows that, for every cut $\theta^{\prime}$ for $\psi$ in $K$, there is a cut $\theta$ for $\varphi$ in $K$, such that $\theta \subset \theta^{\prime}$. Hence, from Lemma 1, it follows that $\varphi \triangleleft_{K} \psi$. By the construction of $\varphi$ and $\psi$, and since Diff $\left(r, r^{\prime}\right) \cap$ $\operatorname{Diff}(K, r)=\varnothing$, we derive that $\varphi \equiv \cup S S(\varphi) \psi$. Hence, from (EP1) it follows that $\varphi<_{K} \psi$. Finally, the construction of $\varphi$ and $\psi$ entails, also, that $[\neg \varphi]=\{r\}$ and $[\neg \psi]=\left\{r^{\prime}\right\}$, hence from (EF), $r \prec_{K} r^{\prime}$ as desired.

For (Q2), assume that, for any two worlds $r, r^{\prime} \in \mathbb{M}, \operatorname{Diff}(K, r)=\operatorname{Diff}\left(K, r^{\prime}\right)$ and $\operatorname{Diff}\left(r, r^{\prime}\right) \cap \operatorname{Diff}(K, r)=\varnothing$. We need to show that $r \approx_{K} r^{\prime}$. If $\operatorname{Diff}(K, r)=\mathcal{P}$, then $r=r^{\prime}$ and, therefore, (Q2) trivially holds. Moreover, if $\operatorname{Diff}(K, r)=\varnothing$, then $r, r^{\prime} \in[K]$. Then, the faithfulness of $\preceq_{K}$ entails (Q2). Assume, therefore, that $\varnothing \neq \operatorname{Diff}(K, r) \subset \mathcal{P}$.

Construct the sentence $\psi$ as follows: $\psi=\bigvee \overline{r^{\prime}}$. Clearly, $K \models \psi$ and $r^{\prime} \models \neg \psi$. Moreover, by construction, the only cut $\theta^{\prime}$ for $\psi$ in $K$ is the set of all units of $K$, that can be expressed

in $\mathcal{L}^{\text {Diff }\left(K, r^{\prime}\right)}$. Now, construct the sentence $\varphi$ as follows: $\varphi=\bigvee \bar{r}$. Clearly, $K \models \varphi$ and $r \mid=\neg \varphi$. Moreover, by construction, the only cut for $\varphi$ in $K$ is the set of all units of $K$, that can be expressed in $\mathcal{L}^{\text {Diff(K,r) }}$. Since Diff $(K, r)=\operatorname{Diff}\left(K, r^{\prime}\right)$, it follows that $\theta=\theta^{\prime}$ (i.e., the set of all cuts for $\varphi$ in $K$ is equal to the set of all cuts for $\psi$ in $K$ ). Hence, from Lemma 2, it follows that $\varphi \sim_{K} \psi$. By the construction of $\varphi$ and $\psi$, and since Diff $\left(r, r^{\prime}\right) \cap$ $\operatorname{Diff}(K, r)=\varnothing$, we derive that $\varphi \equiv_{\cup S S(\varphi)} \psi$. Hence, from (EP2) it follows that $\varphi \simeq_{K} \psi$. Finally, the construction of $\varphi$ and $\psi$ entails, also, that $[\neg \varphi]=\{r\}$ and $[\neg \psi]=\left\{r^{\prime}\right\}$, hence from $(\mathrm{EF}), r \approx_{K} r^{\prime}$ as desired.

\subsection{A Note on Safe and Kernel Contraction}

Safe contraction is one of the important alternatives to partial-meet contraction (Alchourrón \& Makinson, 1985). Contrary to the latter which is based on a selection of sentences to be retained, the former is based on a selection of sentences to be removed. In safe contraction of a belief set $K$ by a sentence of the language, an acyclical hierarchy (i.e., a special kind of relation) over the sentences of $K$ determines which sentences ought to be discarded from it.

Any safe-contraction function for a belief set $K$ is, also, a partial-meet contraction function for $K$; the converse in not true. Nevertheless, it turns out that, if certain restrictions are imposed on hierarchies, the induced subclass of safe-contraction functions coincides with the family of transitively relational partial-meet contraction functions; i.e., functions that

18. To see that $K \models \psi$, observe that $[\neg \psi]=\left\{r^{\prime}\right\}$. However, $[\psi]=\mathbb{M}-[\neg \psi]$, therefore, $[\psi]=\mathbb{M}-\left\{r^{\prime}\right\}$. From $\varnothing \neq[K] \subseteq \mathbb{M}$ and $r^{\prime} \notin[K]$, we derive that $[K] \subseteq[\psi]$. In other words, all $K$-worlds are $\psi$-worlds, which implies that $K \models \psi$. 
satisfy the AGM postulates for contraction (Alchourrón \& Makinson, 1986). ${ }^{19}$ Furthermore, an hierarchy over $K$ can be regarded as an epistemic-entrenchment preorder with respect to $K$, and vice versa (Gärdenfors, 1988). Therefore, our results on the epistemic-entrenchment characterization of Parikh's axiom (P) can, also, be applied to identify relevance-sensitive revision functions that correspond (via Levi identity) to safe contractions.

A natural non-relational generalization of safe contraction, as well as a generalization of partial-meet contraction, is called kernel contraction (Hansson, 1994). Although kernel contraction is usually associated with the contraction of belief bases, it can be applied to belief sets as well; herein, we shall confine ourselves to kernel contraction of (consistent) belief bases.

For presenting the process of kernel (base) contraction, let $B$ be a belief base, and let $\varphi$ be a sentence of $\mathcal{L}$. A $\varphi$-kernel of $B$ is a minimal subset of $B$ that implies $\varphi$. The set of all $\varphi$-kernels of $B$ is called kernel set of $B$ modulo $\varphi$, and it is denoted by $B \Perp \varphi$. An incision function $\sigma$ for $B$ is a function that takes as argument a kernel set $B \Perp \varphi$ (for all $\varphi \in \mathcal{L}$ ), and selects at least one sentence (makes an incision) of each $\varphi$-kernel of $B$ to be deleted.

An incision function $\sigma$ for $B$ gives rise to a kernel-contraction function $\dot{-}_{\sigma}$ (for $B$ ), by means of the following condition:

$$
B \dot{\circ} \sigma \varphi=B-\sigma(B \Perp \varphi) \text {. }
$$

Clearly, the kernel contraction of $B$ by $\varphi, B \dot{ }_{\sigma} \varphi$, is obtained by erasing from $B$ the sentences selected by the incision function $\sigma$.

As earlier stated, minimal support sets and cuts are in close relation with kernels and incision functions, respectively. Nevertheless, there are two crucial differences. Firstly, incision functions are not necessarily minimal, while cuts always are by definition. Note that an incision function $\sigma$ for a belief base $B$ is minimal iff no proper subset of $\sigma(B \Perp \varphi)$ defines an incision function (Falappa, Fermé, \& Kern-Isberner, 2006). Secondly, and most importantly, minimal support sets and cuts refer only to units (of a theory), while Hansson's concepts refer to arbitrary sentences (of a belief base). The aforementioned remarks suffice to make kernels and incision functions non-relevance-sensitive in general, in contrast with minimal support sets and cuts.

It has been pointed out by Falappa et al. (2006) that kernel-contraction functions (for belief bases) induced by minimal incision functions are, essentially, maxichoice (base) contraction functions - a special type of contraction functions that generate "too large" belief bases. In case the unit set of a theory $K$ is considered to be a belief base $B$ for $K$ (i.e., $K=C n(B))$, the minimal support sets and cuts of $K$ correspond precisely to the kernels and minimal incision functions of $B$, respectively. This, in turn, implies an interesting correlation between cuts of $K$ and maxichoice contraction functions for $B$.

Furthermore, given that the belief base $B$ is the unit set of the theory $K, B$ "splits" into disjoint ("refined") compartments, through the finest $K$-splitting. It is not hard to verify, then, that kernel-contraction functions for $B$ are relevance-sensitive. To see this, suppose, without loss of generality, that $B=\left\{\chi_{1}, \chi_{2}, \chi_{3}\right\}$ (where $\chi_{1}, \chi_{2}$ and $\chi_{3}$ are syntax-irrelevant sentences), and consider any sentence $\varphi$ of $\mathcal{L}$, such that $\mathcal{L}_{\varphi} \subseteq \mathcal{L}_{\chi_{1}} \cup \mathcal{L}_{\chi_{2}}$. Obviously, the

19. The AGM postulates for contraction are the counterpart of postulates $(K * 1)-(K * 8)$ in the realm of contraction (Alchourrón et al., 1985). 
sentences of all $\varphi$-kernels of $B$ cannot contain $\chi_{3}$. Since an incision function for $B$ selects sentences of each $\varphi$-kernels, the sentence $\chi_{3}$ will not be affected by the kernel contraction.

Undoubtedly, a thorough investigation of the way that kernel contraction, and its specialization safe contraction, relate to Parikh's notion of relevance constitutes a compelling avenue for future research. ${ }^{20}$

\section{Epistemic-Entrenchment Characterization of (P2)}

The epistemic-entrenchment characterization of condition (P2) is introduced in this section.

\subsection{Initial Considerations}

Definition 16 (Epistemic-Entrenchment Preorder Filtering). Let $K$ be a theory of $\mathcal{L}$. For an epistemic-entrenchment preorder $\leq_{K}$ and a contingent sentence $x \in \mathcal{L}$, the $x$-filtering of $\leq_{K}$, denoted by $\leq_{K}^{x}$, is as follows:

$$
\begin{gathered}
\varphi \leq_{K}^{x} \psi \quad \text { iff } \quad \text { there is } a \nu \in C n_{\mathcal{L}_{x}}(x), \text { with } \nu \models \psi \text {, such that for all } \mu \in C n_{\mathcal{L}_{x}}(x), \\
\text { with } \mu \models \varphi, \mu \leq_{K} \nu .
\end{gathered}
$$

In the above definition, $\varphi, \psi$ are any arbitrary contingent sentences of $K$. It is not hard to verify that, for any contingent sentence $x \in \mathcal{L}$, if $\leq_{K}$ is a total preorder, then so is $\leq_{K}^{x}$. Furthermore, if $\mathcal{L}_{x}=\mathcal{L}$, then it follows from postulate (EE2) (Definition 2) that $x$-filtering has no effect on $\leq_{K}$; i.e., $\leq_{K}^{x}=\leq_{K}$. Note, lastly, that $\leq_{K}^{x}$ depends entirely on $\mathcal{L}_{x}$, as in the case of $\preceq_{K}^{x}$ (Section 6). That is to say, if $x, z$ are two sentences such that $\mathcal{L}_{x}=\mathcal{L}_{z}$, then $\leq_{K}^{x}=\leq_{K}^{z}$, even if $x \not \equiv z$.

\subsection{Characterization Condition}

Now we can formulate the epistemic-entrenchment characterization of condition (P2), which is condition (EP3) below (the preorders $\leq_{K}$ and $\leq_{C n(x)}$ are associated with $K$ and $C n(x)$, respectively):

(EP3) If $K=C n(x, y)$ and $\mathcal{L}_{x} \cap \mathcal{L}_{y}=\varnothing$, then $\leq_{K}^{x}=\leq_{C n(x)}^{x}$.

Condition (EP3) is, essentially, a natural constraint on the relative "retractability" of sentences, and it is in the spirit of (Q3) making associations between epistemic-entrenchment preorders associated with different (overlapping) theories.

\subsection{Characterization Result}

Theorem 7 shows that (EP3) characterizes precisely the family of epistemic-entrenchment preorders corresponding to AGM revision functions satisfying condition (P2).

Theorem 7. Let $*$ be a revision function that satisfies $(K * 1)-(K * 8)$, and let $\left\{\leq_{K}\right\}_{K \in \mathbb{K}}$ be a family of epistemic-entrenchment preorders (one for each theory $K$ of $\mathcal{L}$ ), corresponding to $*$ by means of (E*). Then, $*$ satisfies (P2) iff $\left\{\leq_{K}\right\}_{K \in \mathbb{K}}$ satisfies (EP3).

20. A particular type of local kernel contraction has been studied by Hansson and Wassermann (2002). 


\section{Proof.}

$(\Rightarrow)$

Assume that $*$ satisfies $(\mathrm{P} 2)$. Let $K, H$ be any two theories of $\mathcal{L}$, such that, for some sentences $x, y \in \mathcal{L}, K=C n(x, y), H=C n(x)$, and $\mathcal{L}_{x} \cap \mathcal{L}_{y}=\varnothing$. First, we show that $\leq_{K}^{x} \subseteq \leq_{H}^{x}$.

Let $\varphi, \psi$ be any two contingent sentences of $\mathcal{L}$, such that $\varphi \leq_{K}^{x} \psi$. From the definition of $\leq_{K}^{x}$, we derive that there is a $\nu \in C n_{\mathcal{L}_{x}}(x)$, with $\nu \models \psi$, such that for all $\mu \in C n_{\mathcal{L}_{x}}(x)$, with $\mu \models \varphi, \mu \leq_{K} \nu$. Define $\omega$ to be the sentence $\omega=(\neg \mu) \vee(\neg \nu)$. Clearly, $\omega \in \mathcal{L}_{x}$. This entails that $\mu \notin K * \omega$, and then by (P2), $\mu \notin C n(x) * \omega$. Hence, there is a $\nu \in C n_{\mathcal{L}_{x}}(x)$, with $\nu \models \psi$, such that for all $\mu \in C n_{\mathcal{L}_{x}}(x)$, with $\mu=\varphi, \mu \leq_{C n(x)} \nu$. This again entails that $\varphi \leq_{C n(x)}^{x} \psi$. Consequently, $\leq_{K}^{x} \subseteq \leq_{H}^{x}$ as desired. $(\Leftarrow)$

The proof of the converse, i.e., $\leq_{H}^{x} \subseteq \leq_{K}^{x}$, is totally symmetric.

Assume that (EP3) is satisfied. Let $K$ be any theory of $\mathcal{L}$, such that, for some sentences $x, y \in \mathcal{L}, K=C n(x, y)$ and $\mathcal{L}_{x} \cap \mathcal{L}_{y}=\varnothing$. Let $\omega$ be any contingent sentence in $\mathcal{L}_{x}$. First, we show that $(C n(x) * \omega) \cap \mathcal{L}_{x} \subseteq(K * \omega) \cap \mathcal{L}_{x}$.

Consider any $\mu \in K \cap \mathcal{L}_{x}$, such that $\mu \notin(K * \omega) \cap \mathcal{L}_{x}$. Hence $\mu \leq_{K} \nu$, for some $\nu \in K \cap \mathcal{L}_{x} \cdot{ }^{21}$ From the definition of $\leq_{K}^{x}$, we derive that $\varphi \leq_{K}^{x} \psi$, if $\varphi$ is defined to be the disjunction of all these $\mu$, and if $\psi$ is defined to be logically equivalent to (one of these) $\nu$. Then from (EP3), it follows that $\varphi \leq_{C n(x)}^{x} \psi$. Hence, from the definition of $\leq_{C n(x)}^{x}$, we derive that there is a $\nu^{\prime} \in C n_{\mathcal{L}_{x}}(x)$, with $\nu^{\prime} \models \psi$, such that for all $\mu \in C n_{\mathcal{L}_{x}}(x)$, with $\mu=\varphi, \mu \leq_{C n(x)} \nu^{\prime}$. This again entails that $\mu \notin C n(x) * \omega .{ }^{22}$ Consequently, we have shown that $(C n(x) * \omega) \cap \mathcal{L}_{x} \subseteq(K * \omega) \cap \mathcal{L}_{x}$.

The proof of the converse, i.e., $(K * \omega) \cap \mathcal{L}_{x} \subseteq(C n(x) * \omega) \cap \mathcal{L}_{x}$, is totally symmetric.

\subsection{Combining the Results}

Putting together the results of Theorems 6 and 7, we obtain immediately the following theorem that provides the epistemic-entrenchment characterization of (the strong version of) Parikh's relevance-sensitive axiom.

Theorem 8. Let $*$ be a revision function that satisfies $(K * 1)-(K * 8)$, and let $\left\{\leq_{K}\right\}_{K \in \mathbb{K}}$ be a family of epistemic-entrenchment preorders (one for each theory $K$ of $\mathcal{L}$ ), corresponding to $*$ by means of $(E *)$. Then, $*$ satisfies strong $(P)$ iff $\left\{\leq_{K}\right\}_{K \in \mathbb{K}}$ satisfies (EP1)-(EP3).

Proof. Follows immediately from Theorems 6 and 7.

\subsection{A Note on Ensconcement-Based Revision}

At this point, we make some observations that are crucial for a potential implementation of an effective AGM belief-revision system for real-world applications, where a major obstacle is the large amount of information that, in principle, the user needs to provide to the system

21. Note that if $\nu \notin(K * \omega) \cap \mathcal{L}_{x}$, then $\mu$ and $\nu$ are equally entrenched in $K$.

22. To see this, observe that in order to accommodate $\omega$ in $C n(x)$, either $\mu$ or $\nu$ has to be removed from it (or both, in case they are equally entrenched in $C n(x)$ ). However, taking into account that $\psi \equiv \nu$ by definition, if we give up $\nu$, we also have to remove $\nu^{\prime}$ from $C n(x)$ (for otherwise, logical closure will bring $\nu^{\prime}$ back, since $\nu^{\prime} \models \psi$ ). Hence, from $\mu \leq_{C n(x)} \nu^{\prime}$, we have to give up $\mu$; that is, $\mu \notin C n(x) * \omega$. 
(Williams \& Sims, 2000). ${ }^{23}$ Recall that, in order to construct an AGM revision function (encoding a particular revision policy), a rational agent must be equipped with a family of (faithful or epistemic-entrenchment or selection-function) preorders; i.e., one preorder for every theory of $\mathcal{L}$. The problem is that the size of each one of these preorders is, in general, exponential to the number of propositional variables in the language.

The epistemic-entrenchment characterization of axiom $(\mathrm{P})$ - contrary to its faithfulpreorders characterization provided by Peppas et al. (2015) - is better aligned with ensconcement-based revision (Williams, 1992, 1993, 1994), a computational approach in the context of belief base revision schemes (Nebel, 1998). ${ }^{24}$ Briefly, an ensconcement is a total preorder $\sqsubseteq_{B}$ over the elements (sentences) of a belief base $B$, that can be "blown up" to a full epistemic-entrenchment preorder $\leq_{C n(B)}$, related to $C n(B)$. In other words, it is a (typically) concise representation of an epistemic-entrenchment preorder, since the size of an ensconcement is linear to the size of the knowledge base $B$.

An ensconcement ordering $\sqsubseteq_{B}$ satisfies the following conditions: ${ }^{25}$

(巨1) For all non-tautological sentences $\varphi$ in $B,\left\{\psi \in B: \varphi \sqsubset_{B} \psi\right\} \not \models \varphi$.

(巨2) For all $\varphi \in B, \varphi$ is a tautology iff $\psi \sqsubseteq_{B} \varphi$, for all $\psi \in B$.

Furthermore, $\sqsubseteq_{B}$ satisfies the following priority consistency condition, reported in (Rott, 1991a):

(PCC) For all $\varphi \in B$, if $B^{\prime}$ is a non-empty subset of $B$ that entails $\varphi$, then there is a $\psi \in B^{\prime}$, such that $\psi \sqsubseteq_{B} \varphi$.

Condition (PCC) is a necessary and sufficient condition for the extension of any total preorder $\sqsubseteq_{B}$ to an epistemic-entrenchment preorder $\leq_{C n(B)}$ on $C n(B)$ (Rott, 1991a). As a consequence, ensconcement orderings are always extensible to epistemic-entrenchment preorders. $^{26}$

Having characterized axiom $(\mathrm{P})$ in terms of epistemic entrenchments, in the next two sections, we proceed to the characterization of conditions (P1) and (P2) in terms of the partial-meet model.

\section{Partial-Meet Characterization of (P1)}

This section introduces the partial-meet characterization of condition (P1).

23. A recent attempt implementing the AGM belief-revision process, in the context of Answer Set Programming, has been made by Aravanis and Peppas (2017).

24. Another promising approach towards the direction of making the belief-revision process tractable is Parametrized Difference revision (Peppas \& Williams, 2018; Aravanis, Peppas, \& Williams, 2019).

25. $\sqsubset_{B}$ denotes the strict part of $\sqsubseteq_{B}$.

26. Williams $(1993,1994)$ provided an explicit construction of such an extension. Note, moreover, that an axiomatic characterization for ensconcement-based contraction functions was provided by Fermé, Krevneris, and Reis (2008). 


\subsection{Initial Considerations}

Before formulating the partial-meet characterization of (P1), we need some important remarks. Let $K$ be a theory of $\mathcal{L}$, and let $\varphi$ be a sentence, such that $\neg \varphi \in K$. Grove (1988) pointed out that there is a one-to-one correspondence between the $\neg \varphi$-remainders of $K$ and the $\varphi$-worlds. That is to say, every $\varphi$-world $r$ relates to a single $\neg \varphi$-remainder $R$ of $K$, and, conversely, every $\neg \varphi$-remainder $R$ of $K$ relates to a single $\varphi$-world $r$. From now on, we shall denote by $r_{R}$ the $\varphi$-world that corresponds to the $\neg \varphi$-remainder $R$ of $K$.

Definition 17 (Cell Sentence). Let $K$ be a theory of $\mathcal{L}$, and let $\varphi$ be a sentence, such that $\neg \varphi \in K$. Moreover, let $R$ be a $\neg \varphi$-remainder of $K$, and let $r_{R}$ be the $\varphi$-world that corresponds to $R$. We shall refer to the sentence $\sigma_{R}=\bigvee \overline{r_{R}}$ as the cell sentence of $R$.

By construction, $\left[\neg \sigma_{R}\right]=\left\{r_{R}\right\}$ and $\sigma_{R} \in K{ }^{27}$ The withdrawal of $\sigma_{R}$ from $K$ imposes that, at least, one $\neg \sigma_{R}$-world must be (set-theoretically) added to $[K]$. However, the only such world is $r_{R}$, hence, the only $\sigma_{R}$-remainder of $K$ is the set $R$; i.e., $K \perp \sigma_{R}=\{R\}$.

Then, for any two $\neg \varphi$-remainders $R, R^{\prime}$ of $K$, and their corresponding cell sentences $\sigma_{R}, \sigma_{R^{\prime}} \in K$, condition (EP) of Section 3.5 is reformulated to condition (EP)' as follows:

$$
(\mathbf{E P})^{\prime} \quad \sigma_{R^{\prime}} \leq_{K} \sigma_{R} \quad \text { iff } \quad R \leqq_{K} R^{\prime} .
$$

Condition (EP)', essentially, says that the epistemic value of a $\neg \varphi$-remainder of $K$ is inversely proportional to the epistemic value of the corresponding cell sentence of the $\neg \varphi$ remainder (with respect to $K$ ).

\subsection{Characterization Conditions}

With the aforementioned observations, we formulate the partial-meet duplicates of (EP1) and (EP2), which are conditions (PM1) and (PM2), respectively, presented below: ${ }^{28}$

(PM1) If $\sigma_{R} \triangleleft_{K} \sigma_{R^{\prime}}$ and $\sigma_{R} \equiv \cup S S\left(\sigma_{R}\right) \sigma_{R^{\prime}}$, then $R^{\prime} \lessdot_{K} R$.

(PM2) If $\sigma_{R} \sim_{K} \sigma_{R^{\prime}}$ and $\sigma_{R} \equiv \cup S S\left(\sigma_{R}\right) \sigma_{R^{\prime}}$, then $R^{\prime} \cong_{K} R$.

There is, obviously, a strong resemblance between conditions (PM1)-(PM2) and (EP1)(EP2). This is no accident; it is due to the close relation between a $\neg \varphi$-remainder $R$ of $K$ and the cell sentence $\sigma_{R} \in K$, pointed out earlier.

\subsection{Characterization Result}

Theorem 9 shows that (PM1) and (PM2) characterize precisely the family of selectionfunction preorders corresponding to AGM revision functions satisfying condition (P1).

Theorem 9. Let $*$ be a revision function that satisfies $(K * 1)-(K * 8)$, and let $\left\{\leqq_{K}\right\}_{K \in \mathbb{K}}$ be a family of selection-function preorders (one for each theory $K$ of $\mathcal{L}$ ), corresponding to * by means of (PM*). Then, * satisfies (P1) iff $\left\{\leqq_{K}\right\}_{K \in \mathbb{K}}$ satisfies (PM1)-(PM2).

Proof. Follows immediately from Theorem 6 and condition $(\mathrm{EP})^{\prime}$.

27. See Footnote 18 in the proof of Theorem 6 for the justification.

28 . $\lessdot_{K}$ and $\cong_{K}$ denote the strict and symmetric part of $\leqq_{K}$, respectively. 


\section{Partial-Meet Characterization of (P2)}

Lastly, we turn to the partial-meet characterization of condition (P2).

\subsection{Initial Considerations}

As in the case of faithful-preorders and epistemic-entrenchment characterizations of (P2), one more definition is in order.

Definition 18 (Selection-Function Preorder Filtering). Let $K$ be a theory of $\mathcal{L}$, and let $\varphi$ be a sentence, such that $\neg \varphi \in K$. Moreover, let $\leqq_{K}$ be a selection-function preorder, and let $\leq_{K}$ be the epistemic-entrenchment preorder that corresponds to the revision function associated with $\leqq_{K}$ at $K$ (via condition (EP)). Then, for a contingent sentence $x \in \mathcal{L}$, the $x$-filtering of $\leqq_{K}$, denoted by $\leqq_{K}^{x}$, is as follows:

$$
R^{\prime} \leqq_{K}^{x} R \quad \text { iff } \quad \sigma_{R} \leq_{K}^{x} \sigma_{R^{\prime}}
$$

In the above definition, $R, R^{\prime}$ are any arbitrary $\neg \varphi$-remainders of $K$, and $\sigma_{R}, \sigma_{R^{\prime}}$ are their corresponding cell sentences. It is not hard to verify that, for any contingent sentence $x \in \mathcal{L}$, if $\leq_{K}^{x}$ is a total preorder, then so is $\leqq_{K}^{x}$. Moreover, if $\mathcal{L}_{x}=\mathcal{L}$, then condition $(\mathrm{EP})^{\prime}$ implies that $x$-filtering has no effect on $\leqq_{K}$; i.e., $\leqq_{K}^{x}=\leqq_{K}$.

\subsection{Characterization Condition}

It turns out that the partial-meet duplicate of (EP3) is the following condition:

(PM3) If $K=C n(x, y)$ and $\mathcal{L}_{x} \cap \mathcal{L}_{y}=\varnothing$, then $\leqq_{K}^{x}=\leqq_{C n(x)}^{x}$.

Again, condition (PM3) is in the spirit of (EP3) making associations between selectionfunction preorders related to different (overlapping) theories as well.

\subsection{Characterization Result}

Theorem 10 shows that (PM3) characterizes precisely the family of selection-function preorders corresponding to AGM revision functions satisfying condition (P2).

Theorem 10. Let $*$ be a revision function that satisfies $(K * 1)-(K * 8)$, and let $\left\{\leqq_{K}\right\}_{K \in \mathbb{K}}$ be a family of selection-function preorders (one for each theory $K$ of $\mathcal{L}$ ), corresponding to * by means of (PM*). Then, * satisfies (P2) iff $\left\{\leqq_{K}\right\}_{K \in \mathbb{K}}$ satisfies (PM3).

Proof. Follows immediately from Theorem 7 and the definition of $\leqq_{K}^{x}$ (Definition 18).

\subsection{Combining the Results}

Putting together the results of Theorems 9 and 10, we obtain immediately the following theorem that provides the partial-meet characterization of (the strong version of) Parikh's relevance-sensitive axiom. 
Theorem 11. Let $*$ be a revision function that satisfies $(K * 1)-(K * 8)$, and let $\left\{\leqq_{K}\right\}_{K \in \mathbb{K}}$ be a family of selection-function preorders (one for each theory $K$ of $\mathcal{L}$ ), corresponding to * by means of $(P M *)$. Then, $*$ satisfies strong $(P)$ iff $\left\{\leqq_{K}\right\}_{K \in \mathbb{K}}$ satisfies (PM1)-(PM3).

Proof. Follows immediately from Theorems 9 and 10.

\section{Conclusion}

Parikh's relevance-sensitive axiom (P) constitutes an essential axiom for rational belief change, supplementing the AGM postulates for revision. This is true, not only from a theoretical viewpoint, but also from the perspective of a successful implementation of an AGM belief-revision system, for real-world applications. Assuming that the revision task grows exponentially more difficult (in the worst case) with an increase in the size of the knowledge base, one way to attack the problem is to try to reduce the size of the set to be revised.

In this article, we completed the constructive view of axiom $(\mathrm{P})$, providing its epistemicentrenchment and partial-meet characterizations. In particular, we introduced conditions (EP1)-(EP3) and (PM1)-(PM3), which are natural constraints on the relative "retractability" of sentences and remainders, respectively. Conditions (EP1)-(EP3) and (PM1)-(PM3) characterize precisely the class of epistemic-entrenchment and that of selection-function preorders, respectively, corresponding to AGM revision functions satisfying (the strong version of) axiom (P). Note that condition (EPC), which is equivalent to (EP1)-(EP2) in the special case of complete belief sets, has been independently motivated and studied in Belief Revision.

Future work is to be devoted to a more concrete account of relevance in Belief Revision. Steps towards this direction have been made by Delgrande and Peppas (2018), who generalized Parikh's axiom $(\mathrm{P})$, as relevance was considered to be a context-dependent property of the agent's underlying belief state, going beyond the structure of a belief set.

The notion of language splitting seems intrinsic to any attempt to form a theory of anything at all. The assumption that we can ignore some aspects, while considering others, is inherent in almost all intellectual activity. As a consequence, the results reported herein could, also, be significant for related domains of Artificial Intelligence, where the notions of relevance and local change play a vital role (both conceptually and computationally), such as multi-agent systems revision, ontology update and merging, cognitive modelling, heuristic search and optimization.

\section{Acknowledgments}

This article is a substantial extension and elaboration of previous work, published by Aravanis, Peppas, and Williams (2017). Part of the work was carried out while Pavlos Peppas was, also, affiliated with the School of Computer Science, at the University of Technology Sydney. We are grateful to the anonymous reviewers for their valuable and constructive comments. 


\section{References}

Alchourrón, C., Gärdenfors, P., \& Makinson, D. (1985). On the logic of theory change: Partial meet contraction and revision functions. Journal of Symbolic Logic, 50(2), $510-530$.

Alchourrón, C., \& Makinson, D. (1985). On the logic of theory change: Safe contractions. Studia Logica, 44, 405-422.

Alchourrón, C., \& Makinson, D. (1986). Maps between some differents kinds of contraction functions: The finite case. Studia Logica, 45, 187-198.

Aravanis, T., Peppas, P., \& Williams, M.-A. (2017). Epistemic-entrenchment characterization of Parikh's axiom. In Proceedings of the 26th International Joint Conference on Artificial Intelligence, IJCAI 2017, pp. 772-778.

Aravanis, T., Peppas, P., \& Williams, M.-A. (2019). An investigation of parametrized difference revision operators. Annals of Mathematics and Artificial Intelligence, Springer.

Aravanis, T. I., \& Peppas, P. (2017). Belief revision in Answer Set Programming. In Proceedings of the 21st Pan-Hellenic Conference on Informatics, PCI 2017, pp. 2:12:5, New York, NY, USA. ACM.

Chopra, S., Georgatos, K., \& Parikh, R. (2001). Relevance sensitive non-monotonic inference on belief sequences. Journal of Applied Non-Classical Logics, 11(1-2), 131-150.

Chopra, S., \& Parikh, R. (2000). Relevance sensitive belief structures. Annals of Mathematics and Artificial Intelligence, 28(1-4), 259-285.

Darwiche, A., \& Pearl, J. (1997). On the logic of iterated belief revision. Artificial Intelligence, 89, 1-29.

Delgrande, J., \& Peppas, P. (2018). Incorporating relevance in epistemic states in belief revision. In Proceedings of the 16th International Conference on Principles of Knowledge Representation and Reasoning, KR 2018, pp. 230-239.

Falappa, M. A., Fermé, E. L., \& Kern-Isberner, G. (2006). On the logic of theory change: Relations between incision and selection functions. In Proceedings of the 17th European Conference on Artificial Intelligence, ECAI 2006, pp. 402-406.

Fermé, E., Krevneris, M., \& Reis, M. (2008). An axiomatic characterization of ensconcement-based contraction. Journal of Logic and Computation, 18(5), 739-753.

Gärdenfors, P. (1988). Knowledge in Flux - Modeling the Dynamics of Epistemic States. MIT Press, Cambridge, Massachusetts.

Gärdenfors, P. (1990). Belief revision and relevance. In PSA: Proceedings of the Biennial Meeting of the Philosophy of Science Association, pp. 349-365.

Gärdenfors, P., \& Makinson, D. (1988). Revisions of knowledge systems using epistemic entrenchment. In Proceedings of the 2nd Conference on Theoretical Aspects of Reasoning About Knowledge, pp. 83-95, Pacific Grove, California. Morgan Kaufmann.

Grove, A. (1988). Two modellings for theory change. Journal of Philosophical Logic, 17(2), $157-170$. 
Hansson, S. O. (1994). Kernel contraction. Journal of Symbolic Logic, 59, 845-859.

Hansson, S. O., \& Wassermann, R. (2002). Local change. Studia Logica: An International Journal for Symbolic Logic, 70(1), 49-76.

Katsuno, H., \& Mendelzon, A. (1991). Propositional knowledge base revision and minimal change. Artificial Intelligence, 52(3), 263-294.

Kern-Isberner, G., \& Brewka, G. (2017). Strong syntax splitting for iterated belief revision. In Proceedings of the 26th International Joint Conference on Artificial Intelligence, IJCAI 2017, pp. 1131-1137.

Kourousias, G., \& Makinson, D. (2007). Parallel interpolation, splitting, and relevance in belief change. Journal of Symbolic Logic, 72(3), 994-1002.

Lindström, S., \& Rabinowicz, W. (1991). Epistemic entrenchment with incomparabilities and relational belief revision. In Fuhrmann, A., \& Morreau, M. (Eds.), The Logic of Theory Change, Vol. 465 of Lecture Notes in Artificial Intelligence, pp. 93-126. Springer Berlin Heidelberg.

Makinson, D. (2009). Propositional relevance through letter-sharing. Journal of Applied Logic, 7, 377-387.

Nebel, B. (1998). How hard is it to revise a belief base?. In Dubois, D., \& Prade, H. (Eds.), Handbook of Defeasible Reasoning and Uncertainty Management Systems, Vol. 3: Belief Change, pp. 77-145. Kluwer Academic.

Nebel, B. (1999). Syntax-based approaches to belief revision. In Gärdenfors, P. (Ed.), Belief Revision, Theoretical Computer Science, pp. 52-88. Cambridge University Press.

Parikh, R. (1999). Beliefs, belief revision, and splitting languages. In Moss, L. S., Ginzburg, J., \& de Rijke, M. (Eds.), Logic, Language and Computation, Vol. 2, pp. 266-278. CSLI Publications.

Parikh, R. (2011). Beth definability, interpolation and language splitting. Synthese, 179, $211-221$.

Peppas, P. (2008). Belief revision. In van Harmelen, F., Lifschitz, V., \& Porter, B. (Eds.), Handbook of Knowledge Representation, pp. 317-359. Elsevier Science.

Peppas, P., Foo, N., \& Nayak, A. (2000). Measuring similarity in belief revision. Journal of Logic and Computation, 10, 603-619.

Peppas, P., \& Williams, M.-A. (1995). Constructive modellings for theory change. Notre Dame Journal of Formal Logic, 36(1), 120-133.

Peppas, P., \& Williams, M.-A. (2018). Parametrised difference revision. In Proceedings of the 16th International Conference on Principles of Knowledge Representation and Reasoning, KR 2018, pp. 277-286.

Peppas, P., Williams, M.-A., Chopra, S., \& Foo, N. (2015). Relevance in belief revision. Artificial Intelligence, 229, 126-138.

Perrussel, L., Marchi, J., \& Zhang, D. (2010). Characterizing relevant belief revision operators. In Li, J. (Ed.), AI 2010: Advances in Artificial Intelligence, Vol. 6464 of Lecture Notes in Computer Science, pp. 42-51. Springer Berlin Heidelberg. 
Riana, J., \& Wassermann, R. (2004). Using relevance to speed up inference: Some empirical results. In Advances in Artificial Intelligence - SBIA 2004, pp. 21-30.

Rott, H. (1991a). A nonmonotonic conditional logic for belief revision. Part 1: Semantics and logic of simple conditionals. In Fuhrmann, A., \& Morreau, M. (Eds.), The Logic of Theory Change, Vol. 465 of Lecture Notes in Artificial Intelligence, pp. 135-181. Springer Berlin Heidelberg.

Rott, H. (1991b). Two methods of constructing contractions and revisions of knowledge systems. Journal of Philosophical Logic, 20(2), 149-173.

Spohn, W. (1988). Ordinal conditional functions: A dynamic theory of epistemic states. In Harper, W. L., \& Skyrms, B. (Eds.), Causation in Decision, Belief Change, and Statistics, Vol. 42 of The University of Western Ontario Series in Philosophy of Science, pp. 105-134. Springer Netherlands.

Subramanian, D., Greiner, R., \& Judea Pearl, E. (1997). Relevance. Artificial Intelligence, $97(1-2), 1-402$.

Wassermann, R. (2001a). Local diagnosis. Journal of Applied Non-Classical Logics, 11(1-2), $107-129$.

Wassermann, R. (2001b). On structured belief bases. In Williams, M.-A., \& Rott, H. (Eds.), Frontiers in Belief Revision, Applied Logic Series, pp. 349-367. Springer Netherlands.

Williams, M.-A. (1992). Two operators for theory bases. In Proceedings of the Australian Joint Conference on Artificial Intelligence, pp. 259-265. World Scientific.

Williams, M.-A. (1993). Transmutations of Knowledge Systems. Ph.D. thesis, University of Sydney, Australia.

Williams, M.-A. (1994). On the logic of theory base change. In MacNish, C., Pearce, D., \& Pereira, L. (Eds.), Logics in Artificial Intelligence - European Workshop JELIA 1994, Vol. 838 of Lecture Notes in Artificial Intelligence, pp. 86-105. Springer Berlin Heidelberg.

Williams, M.-A., \& Sims, A. (2000). SATEN: An object-oriented web-based revision and extraction engine. In International Workshop on Nonmonotonic Reasoning, NMR 2000.

Wu, M., \& Zhang, M. (2008). Maximal invariable update operator based on splitting. In Wang, G., Li, T., Grzymala-Busse, J. W., Miao, D., Skowron, A., \& Yao, Y. (Eds.), Rough Sets and Knowledge Technology, Vol. 5009 of Lecture Notes in Computer Science, pp. 418-425. Springer Berlin Heidelberg. 